\title{
Malware detection using static analysis in Android: a review of FeCO (features, classification, and obfuscation)
}

\author{
Rosmalissa Jusoh ${ }^{\text {Corresp., } 1}{ }^{1}$, Ahmad Firdaus ${ }^{\text {Corresp., } 1}{ }^{1}$, Shahid Anwar ${ }^{2}$, Mohd Zamri Bin Osman ${ }^{1}$, Mohd Faaizie Darmawan \\ ${ }^{3}$, Mohd Faizal Ab Razak ${ }^{1}$ \\ 1 Faculty of Computing, College of Computing and Applied Sciences, Universiti Malaysia Pahang, Pekan, Pahang, Malaysia \\ 2 Department of Software Engineering, University of Lahore, Lahore, Pakistan \\ 3 Faculty of Computer \& Mathematical Sciences, Universiti Teknologi Mara, Tapah, Perak, Malaysia \\ Corresponding Authors: Rosmalissa Jusoh, Ahmad Firdaus \\ Email address: rosmalissa@ump.edu.my, afza85@gmail.com
}

Android is a free open-source operating system (OS), which allows an in-depth understanding of its architecture. Therefore, many manufacturers are utilizing this OS to produce mobile devices (smartphones, smartwatch, and smart glasses) in different brands, including Google Pixel, Motorola, Samsung, and Sony. Notably, the employment of OS leads to a rapid increase in the number of Android users. However, unethical authors tend to develop malware in the devices for wealth, fame, or private purposes. Although practitioners conduct intrusion detection analyses, such as static analysis, there is an inadequate number of review articles discussing the research efforts on this type of analysis. Therefore, this study discusses the articles published from 2009 until 2019 and analyses the steps in the static analysis (reverse engineer, features, and classification) with taxonomy. Following that, the research issue in static analysis is also highlighted. Overall, this study serves as the guidance for novice security practitioners and expert researchers in the proposal of novel research to detect malware through static analysis. 
1 Malware detection using static analysis in Android: A review of FeCO (Features, 2 Classification, and Obfuscation)

3

4

\section{5}

6

7

${ }^{1}$ Rosmalissa Jusoh, ${ }^{1}$ Ahmad Firdaus, ${ }^{2}$ Shahid Anwar, ${ }^{1}$ Mohd Zamri Osman, ${ }^{3}$ Mohd Faaizie Darmawan, ${ }^{1}$ Mohd Faizal Ab Razak

${ }^{1}$ Faculty of Computing (FKOM), College of Computing and Applied Sciences, Universiti Malaysia Pahang, 26600 Pekan, Pahang, Malaysia

${ }^{2}$ Department of Software Engineering, The University of Lahore, 54590, Lahore, Pakistan ${ }^{3}$ Faculty of Computer \& Mathematical Sciences, Universiti Teknologi MARA, 35400 Perak Branch, Tapah Campus, Malaysia

\section{Corresponding Author:}

${ }^{1}$ Ahmad Firdaus, ${ }^{1}$ Rosmalissa Jusoh

Faculty of Computing (FKOM), College of Computing and Applied Sciences, Universiti Malaysia Pahang, 26600 Pekan, Pahang, Malaysia

Email address: firdausza@ump.edu.my, rosmalissa@ump.edu.my

\section{Abstract}

Android is a free open-source operating system (OS), which allows an in-depth understanding of its architecture. Therefore, many manufacturers are utilizing this OS to produce mobile devices (smartphones, smartwatch, and smart glasses) in different brands, including Google Pixel, Motorola, Samsung, and Sony. Notably, the employment of OS leads to a rapid increase in the number of Android users. However, unethical authors tend to develop malware in the devices for wealth, fame, or private purposes. Although practitioners conduct intrusion detection analyses, such as static analysis, there is an inadequate number of review articles discussing the research efforts on this type of analysis. Therefore, this study discusses the articles published from 2009 until 2019 and analyses the steps in the static analysis (reverse engineer, features, and classification) with taxonomy. Following that, the research issue in static analysis is also highlighted. Overall, this study serves as the guidance for novice security practitioners and expert researchers in the proposal of novel research to detect malware through static analysis.

Keywords: Android; Malware; Review; Static analysis; Machine learning; Features

\section{Introduction}

Mobile devices, such as smartphones, iPads, and computer tablets, have become everyday necessities to perform important tasks, including education, paying bills online, bank transactions, 
40 job information, and leisure. Based on the information from an online mobile device production 41 website, Android is one of the popular operating systems (OS) used by manufacturers [1][2]. The 42 open-source platform in Android has facilitated the smartphone manufacturers in producing 43 Android devices of various sizes and types, such as smartphones, smartwatches, smart televisions, 44 and smart glasses. In the most recent decades, the quantity of remarkable Android gadgets accessible worldwide has increased from 38 in 2009 to over 20,000 in 2016 [3]. As a result of the demand for this Android OS, the recent statistics from Statista revealed that the number of Android malware increase to 26.6 million in March 2018 [4]. Moreover, McAfee discovered a malware known as Grabos, which compromises the Android and breaches Google Play Store security [5]. It was also predicted that 17.5 million Android smartphones had downloaded this Grabos mobile malware before they were taken down.

Mobile malware is designed to disable a mobile device, allow malicious acts to remotely control the device, or steal personal information [6]. Moreover, these malicious acts able to run stealthily and bypass permission if the Android kernel is compromised by mobile malware [7][8]. In September 2019, a total of 172 malicious applications were detected on Google Play Store, with approximately 330 million installations. According to researchers, the malicious components were hidden inside the functional applications. When the applications are downloaded, it leads to the appearance of popup advertisements, which remain appear even when the application was closed [9]. To detect this malware, security practitioners conducting malware analysis, which aims to study the malware characteristics and behaviour. There are dynamic, static, and hybrid analysis.

Table 1 shows comparison for static, dynamic and hybrid analysis done from previous researches. Specifically, dynamic analysis is an analysis, which studies the execution and behaviour of the malware [10][11]. However, dynamic analysis is incapable of identifying several parts of the code operating outside the monitoring range. Besides, provided that the dynamic analysis is a high resource-consuming analysis with a high specification for hardware [10], static analysis is another alternative to detect malware. It is an analysis, which examines malware without executing or running the application. Additionally, this analysis able to identify malware more accurately, which would act under unusual conditions [12]. This is due to static analysis examine overall parts of a program including parts that excluded in dynamic analysis. Furthermore, static analysis is able to detect unknown malware just as dynamic analysis could [13] and requiring low resources.

To integrate the characteristics of the static and dynamic method, three-layer detection model called SAMAdroid has been proposed by [14] which combines static and dynamic characteristics. Mobile Sandbox by [15] which proposed to use the results of static analysis to guide the dynamic analysis and finally realize classification. The hybrid analysis technique is great to help in improving the accuracy, but it also has a major drawback such as the waste of time and space for the huge number of malware samples to be detected and analyzed [16][17]. 
80

81

82

83

84

85

86

87

88

89

90

91

92

93

94

95

96

97

98

99

100

101

102

103

104

105

106

107

108

109

110

111

112

113

114

\section{Survey methodology} administration, and more. current article are as follows: other methods.

Table 2 presents the past review articles on Android, with [18] specifically focusing on features, including static, dynamic, hybrid, and metadata. It summarizes the features preferred researchers in their analysis. Comparatively, this study placed more emphasis on features besides classification and obfuscation. Subsequent reviews, namely [19] and [20], highlighted the survey, taxonomy, challenges, advantages, limitations in the existing research in the Android security area, and the technique in the static analysis research on Android. However, compared to the current review, the aforementioned reviews only presented a few features and information on static analysis. In the Android permission category [21], this article reviewed the issues and existed countermeasures for permission-based Android security. It also incorporated the necessary information related to Android permission, namely permission documentation, over-claim of permission, permission

Notably, our review article featured more aspects besides permission by putting an in-depth focus on static analysis. Meanwhile, the other paper, evaluates three anti-viruses, namely Stowaway, AASandbox, and Droidbox. To assist in the decision regarding the better anti-virus, the aforementioned anti-viruses were separated using static and dynamic analyses, followed by a comparison between one another [7]. Another related review [22], provided the common features only and mentioned less the deep learning part in static analysis. However, the information gained from past research, specifically on features, deep learning and obfuscation was still lacking. In comparison to the previous survey articles listed in the Table 2, the main contributions of the

a) Present the information of static analysis in detail, specifically the dataset, reverse engineer, features, and classification from 2009 until 2019.

b) Review on the latest features in ten categories, namely advertisements libraries, application programming interface (API), apk, dex and xml properties, directory path, commands, a function call, geographic location, manifest file, network address, and codebase.

c) Review the classification section, which includes machine learning, deep learning, graph, and

d) Discuss on open research issues, which include the trends in the static analysis, obfuscation, and the list of all previous static analysis experiments.

The remaining section of this article begins with section two, which presents the methodology of this study into four steps. Section three reviews the existing research on static analysis, which concludes the dataset, reverse engineer, features, and classification. Section four discusses the open research issues in the static analysis, followed by section five, which concludes the review. 


\section{1. Methodology}

118 This section describes the method to retrieve the articles related to malware detection using static 119 analysis for Android. We used Web of Science to run the review, eligibility and exclusion criteria, 120 steps of the review process (identification, screening, eligibility), and data analysis.

121

122

123

124

125

126

127

128

129

130

131

132

133

134

135

136

137

138

139

140

141

142

143

144

145

146

147

148

149

\subsection{Identification}

The review was performed based on the main journal database in the Web of Science (WoS). This database covers more than 256 disciplines with millions of journals regarding the subjects related to network security, computer system, development, and planning. It also stores over 100 years of comprehensive backfile and citation data established by Clarivate Analytics (CA), which are ranked through three separate measures, namely citations, papers, and citations per paper. The search strings in the CA database were "static analysis", "malware", and "Android".

There were 430 records identified through database searching. These journals and conferences are mainly from Computer and Security and IEEE Access, which are listed in Table 3. Collections of the studies that are related to Android malware detection using static analysis in the reference section, where studies take up a small proportion in the primary studies. All the studies related to search terms are taken into account, and the searching range is from January 2009 to December 2019.

\subsection{Screening}

Experiment articles were identified in the static analysis, omitting other unrelated articles. Initially, the searching of articles was specified into a journal article and excluded review articles, books, and conference proceedings. To focus specifically on static analysis, the articles, which combined both static and dynamic analyses, were removed. Another criterion focused on the selection of the articles was the use of English, which therefore removed all non-English articles to avoid any difficulty in translating in the future. The selection of articles took place from 2009 to 2019, totaling the duration to 10 years. This duration was suitable for exploring the evolution of research in security areas. Apart from that, the Android platform was the focus of this study.

\subsection{Eligibility}

Figure 1 depicts the review that process involved four steps; identification, screening, eligibility, and analysis. The review was performed in mid of 2019. Based on previous studies, the process used similar keywords related to malware detection, static analysis, and security. After the identification process, we remove any duplicated articles. During the screening process, we 
150 discover 375 documents and remove a few articles and left 172 articles. This is because the articles were unrelated to the interested area. Lastly, we used 150 articles for review [23].

152

153

154

155

156

157

158

159

160

161

162

163

164

165

166

167

168

169

170

171

172

173

174

175

176

177

178

179

180

181

182

183

184

185

\subsection{Data analysis included}

Then we analyzed the remaining articles, extracted the abstract, and downloaded the full articles. This is to find the appropriate topic in-depth and to have a strong justification for the research. Then, this process organized the topic and subtopic accordingly based on the static analysis. Qualitative analysis was performed based on content analysis to identify issues related to this study.

\section{Static analysis}

Mobile malware compromises Android devices (smartphone, smartwatch, and smart television) for wealth, stealing data, and personal purposes. The examples of mobile malware include root exploit, botnets, worms, and Trojan. To detect malware, most of security practitioners perform two types of analysis; dynamic and static.

Specifically, dynamic analysis is an experiment, which detects malware by executing malware and benign applications to monitor and differentiate their behaviours. However, the monitoring of all behaviours is costly and requires high specifications in terms of device memory, CPU, and storage. Furthermore, the malware is inflicted on a device at a certain time or whenever the attacker decides on it. Accordingly, as the dynamic analysis only monitors behaviours at a certain range of time based on the research period, numerous malware activities outside the research period might be omitted [24][25][26]. Furthermore, dynamic analysis requires a separate and closed virtual environment to run a malware and observe its behaviour on the system. However, an isolated setup dynamic leads to an impractical analysis in the Android platform due to the increase in power and memory consumption. While power and memory are the most concerning constraints of Android devices, static analysis is the alternative for the dynamic analysis.

Static analysis is a category of analysis, which investigates the malware application code and examine full activities in an application within an unlimited range of time, by without executing the application [27]. The main step of static analysis procedure is the reverse engineer process, which retrieves the whole code and further scrutinises the structure and substance within the application [28]-[30]. Therefore, this analysis can examine the overall code with low requirement for memory resources and minimum CPU processes. Additionally, the analysis process is prompt due to the absence of the application. With this analysis, unknown malware is also identified using enhanced detection accuracy through machine learning approaches [31][24]. Table 4 presents the advantages and disadvantages of dynamic and static analyses. 
186

187

188

189

190

191

192

193

194

195

196

197

198

199

200

201

202

203

204

205

206

207

208

209

210

211

212

213

214

215

216

217

218

219

220

221

222

223
A lot of researchers publish their works using static approaches for malware detection on the Android platform. Even in this static approach, in its turn, contains a number of approaches. For example, there are signature-based approach and other approach are depending on detection and classification of the source code. Signature-based detection utilizes its specification by having an information of malware signatures determined and arranged in advance inspection [32]. However, signature-based approach are not able to detect unknown malware even though this approach is a set of features that uniquely differentiate the executable code [33].

Obfuscation is one of the obstacles in the static analysis, which is used by malware authors in their malicious software to evade the intrusion detection or antivirus system [26]. The examples of the obfuscation methods are renaming the code, adding unnecessary codes, and encrypting the string. Therefore, security practitioners need to overcome obfuscation to increase their detection results. Accordingly, the alternatives performed by the security practitioners are presented in Section 3.2. Table 4 shows that both static and dynamic analyses have similar limitations despite the selection of the ideal features in minimal amount. In detecting malware, features refer to the attributes or elements to differentiate an application, which may either be malware or benign. Security practitioners are faced with obstacles in investigating various features in all types of categories (e.g., permission, API, directory path, and code-based) and the need to simultaneously reduce these features. Notably, determining the ideal features in minimal amount is crucial to enhance the accuracy of the analyses (e.g., the accuracy of the predictive model) and reduce data and model complexity [18].

Figure 2 illustrates the static analysis operation, which consisted of several steps. The first step was the acquirement of the benign and malware datasets in the Android application, each with the (.apk) filename extension. This was followed by the reverse engineering performed on these applications to retrieve the code by extracting a few folders from one apk file, which consisted of nested files with codes (Java or smali). Furthermore, one apk would comprise approximately a thousand lines of codes. Therefore, with a total of 1000 applications in one dataset, the security practitioners were required to scrutinise millions of lines of code. With the completion of the reverse engineering, an analysis would be conducted, which involved features. Features consist of a series of application characteristics for the detection of malware, while classification is an approach used to differentiate between malware and benign (normal) application. The following section thoroughly discusses the static analysis, which specifically begins with a focus on the dataset.

\subsection{Dataset}

Figure 3 illustrates the Android malware dataset from different places. Notably, the majority of the datasets were obtained from universities. The datasets were in the form of an Android

Peer] Comput. Sci. reviewing PDF | (CS-2020:10:54261:2:0:NEW 14 Mar 2021) 
224

225

226

227

228

229

230

231

232

233

234

235

236

237

238

239

240

241

242

243

244

245

246

247

248

249

250

251

252

253

254

255

256

257

258

259

260

261

application package, which was followed by an .apk filename extension. Malgenome [34] is the name of Android malware dataset, which was made to be publicly available with permission from their administrator. These malware samples, which were collected by North Carolina State University (NCSU) from August 2010 to October 2011, covered multiple families of malware consisting of botnet and root exploit. The characterization of the malware families was based on the method of the installation, the way the malware carried the malicious payloads, and its method of activation.

Androzoo [35] [36] is another dataset consisting of approximately more than three million of Android applications (.apk). This dataset originates from the University of Luxembourg to contribute to the community for research purposes and further explore the notable development in the detection of malware, which damages the Android. Drebin [37] dataset also presents Android malware publicly with strict requirements. A university from Germany (University in Braunschweig, Germany) collected 5560 samples with 179 families. The time range provided for the malware was from August 2010 to October 2012. The university project, which was known as MobileSandbox, was an initiative for the acquirement of samples for academia and industry.

Android malware dataset (AMD) [39] is a public Android malware dataset from the University of South Florida, which consists of 24,650 samples with 71 categorised families. To obtain this dataset, the user is required to acquire permission from the university and provide authentic information with evidence. The academia and the industry are allowed to use these samples for research purposes.

Contagio [40] dataset presents the malware, which focuses on mobile malware, with a condition that the user should omit one sample to obtain another sample. It provides a dropbox for the user to share their mobile malware samples. According to their blogspot [40], the name of the administrator of this dataset is Mila Parkour, who is reachable only through emails. Based on Table 5 , which presents the research articles and the respective datasets, it could be seen that the dataset providers receive significant attention from other universities and the industry. It is hoped that this action would enhance the security of the Android device and its users from time to time.

\subsection{Reverse engineer}

Static analysis is an activity to investigate the code of an application without executing it. In order to investigate, security practitioners implement the reverse engineering method. This method reversed from the executable file to its source code [41]. This reverse engineering process loads the executable into a disassembler to discover what the program does. Figure 4 illustrates the tools used to perform a reverse engineering method, which was also adopted by security practitioners to identify Android malware. Table 6 illustrates the tools adopted in the respective articles. 


\subsection{Features}

264

265

266

267

268

269

270

271

272

273

274

275

276

277

278

279

280

281

282

283

284

285

286

287

288

289

290

Once the researchers reverse engineer the executable file using specific tools, they need to select features from the source code. Feature selection is important in order to increase the accuracy of the detection system. [18], [42], [43]. Figure 5 presents the taxonomy of multiple static features. The next sections are the details for each type of static feature.

\subsubsection{Advertisement libraries}

Provided that most Android applications are available for free download, Android developers need to include advertisement libraries (ad libraries) in the free application for financial purposes. During the run-time of the application, the ad libraries would transfer the data regarding users' activities. The developer would then receive an incentive based on certain metrics of the information. Adrisk [44] scrutinised and measured the risk of the codes of the ad libraries to detect applications, which may harm the users.

\subsubsection{Application Programming Interface}

Application program interface (API) is a set of code ready for certain functionalities. Android application developers use this API for their application. Usually, there is documentation ready to use the API. Nevertheless, the attacker uses certain API for their malware application. Accordingly, to detect malware, security practitioners inspect API features that regularly used by the attackers. The articles that involves API features are Droidlegacy [45], Droidapimiiner [30], Warning system [46], [29], [47], [48], [49], [47], [50], [51], [52], [53], [54], [55], [56], [57], [58], [59], and [60].

\subsubsection{Apk, dex and $x m l$ properties}

Several security practitioners adopted the features, which consist of apk file properties. The authors of the malwares [61] and [62] are examined in two experiments due to the significant number of Android malwares written by a similar person. Therefore, the features of the malwares include serial numbers of the author, author's information, name, contact and organization information, developer certification, author's ID, and public key fingerprints of the author. Other features highlighted in this section are the application name, category, package, description, rating values, rating counts, size, number of zip entries, and common folders [63][64]. 
291

292

293

294

295

296

297

298

299

300

301

302

303

304

305

306

307

308

309

310

311

312

313

314

315

316

317

318

319

320

321

322

323

\subsubsection{Directory path}

Directory path allows access for a specific folder in the operating system (OS). It was found by security practitioners that the attacker incorporated a directory path for a sensitive folder in their malware. Meanwhile, several paths related to Android kernel directory were identified by another study [65], such as 'data/local/tmp/rootshell', '/proc', and '/system/bin/su'.

\subsubsection{Commands}

Two types of commands are available, namely 1) root command and 2) botnet command. Specifically, several root commands were identified by [65] in the Unix machine, such as 'cp', 'cat', 'kill', and 'mount'. Normally, these commands were used by the administrators to execute higher privileged actions in the Unix machine. Provided that Android architecture was based on the Unix kernel, the attackers included root commands in their malware to control the victim's Android devices. Therefore, the identification of root commands is crucial in investigating malwares.

The second type of command is a botnet command. Meanwhile, one type of malware, which is known as a mobile botnet, includes botnet commands in their malware codes, such as 'note', 'push', 'soft', 'window', 'xbox', and 'mark'. The attacker used these commands to communicate with the command and control $(\mathrm{C} \& \mathrm{C})$ server, while droidanalyzer [58] combines API, root command, and botnet command into a set of features to detect root exploit and mobile botnet.

Other than ad libraries, certain researchers inspect the Android Debug Bridge (adb) code. ADB [66] is a tool, which provides a command-line access facility for users or developers to communicate with Android mobile devices. This facility allows the installation of unwanted applications and execution of various Unix by the attacker in the victim's device. Therefore, RODS [67] is a root exploit detection system for the detection of a root exploit malware with ADB features.

\subsubsection{Function call}

In programming, a function call is a declaration, which consists of a name and is followed by an argument in parenthesis. The list of the argument may include any numbers of the name, which are either separated by commas or left empty. Another study by [68] involved the extraction of a function call through readelf, which was then used for the features in machine learning prediction. Meanwhile, [69] extracted the function calls in a graph to identify the nodes from the start to the end of the process. 
324

325

326

327

328

329

330

331

332

333

334

335

336

337

338

339

340

341

342

343

344

345

346

347

348

349

350

351

352

353

354

\subsubsection{Geographic location}

Geographic location is a feature, which identifies the origin of the application. The geographic detector was identified as one of the features in research by [54]. Provided that $35 \%$ of the mobile malware families appeared to originate from China with $40 \%$ of the facilities originating from Russia, Ukraine, Belorus, Latvia, and Lithuania countries, it was crucial to consider geographic location as one of the features for the detection of Android malware. For this reason, researchers increased the risk signal for the applications originating from the aforementioned countries.

\subsubsection{Manifest file}

Android application is built on the top of the application framework which provides an interface for the user. The program is based on the Android application package file in the (.apk) format, which is also used to install an application in android-based mobile devices. It consists of metainf, resource, assets and library directory, classes.dex, resources.arsc, and androidmanifest.xml file. One of the files, androidmanifest.xml (manifest file), is an essential file with contents of various features, such as permission, intent, hardware component, and components of the application (activities, services, broadcast receivers, and content providers) [70].

a) Permission

Permission is a unique security mechanism for Android devices. To enable the permission, the user needs to allow the application during the installation period. However, many users accidentally enable certain permissions, which leads to access to sensitive security-relevant resources. Therefore, permission features were examined in many studies. Based on the application of permission in several studies to measure the risk of the application, permission was further identified as malicious [71], [72]. Some other studies, such as [73][63][74][75][76][77][78][79], used the permission features as the inputs for machine learning prediction.

b) Intent

The intent is coded in the manifest file and allows a component of the application to request certain functionality from another component from other application. For example, application A can use the component of application B for the management of photos in the device despite the exclusion of the component from application A. Provided that this feature enables malicious activities among the attackers, several experiments used intent (declared in the manifest file) as one of the features for the detection of malware, such as [80] and [81].

c) Application component 
355 The manifest file declared application component, which consists of four types, namely 1)

356

357

358

359

360

361

362

363

364

365

366

367

368

369

370

371

372

373

374

375

376

377

378

379

380

381

382

383

384

385

386

387

388 activities, 2) services, 3) broadcast receivers, and 4) content providers. Specifically, activity is represented as the user interface or interactive screen to the users, while service refers to an operation occurring in the backgrounds, which perform the long-service process. This is followed by broadcast receivers, which respond to system-wide broadcast announcements. On the other hand, content providers manage a structured set of application data. Overall, these four components follow a life cycle model during execution. Dexteroid [82] proposed a framework, which systematically guides the event sequences through the reverse engineering/reconstruction of the life cycle models and the extraction of callback sequences from event sequences to detect malicious behaviours.

d) Hardware component

The manifest file also incorporated hardware components in the Android application. To illustrate, the developer requested access to the camera of an Android device by declaring it in the manifest file to enable the use of the camera for the application. However, the attacker declared unrelated hardware components in their game application, such as camera and data. As a result, the security researchers were prompted to use hardware component as the features in their experiment [55] to detect malware [83].

\subsubsection{Network address}

Access to the Internet is essential for attackers to retrieve private information of the victim, change the settings, or execute malicious commands. This process requires the incorporation of the Uniform Resource Locator (URL) or network address in the malware code. The examples of sensitive URLs include the Android Market on Google Play, Gmail, Google calendar, Google documents, and XML schemas. These features were used in [56] and [84] [85] for malware detection.

\subsubsection{Code-based}

Code-based or code structure comprises a line or set of programming language codes in an application. Two studies applied code structures (code chunk grammar) as the features for malware detection, which is focused on the internal structure of the code units [86][87]. This feature enables the analysis and differentiation between malware and benign applications. Another study by [65] identified several code-based strings, namely '.exec', 'forked', 'setptywindowsize', and 'createsubprocess'. In comparison with the normal application, it was found that the attacker frequently used these code-based features in the development of malware. Therefore, these features were also used in this study to detect malware.

Peer] Comput. Sci. reviewing PDF | (CS-2020:10:54261:2:0:NEW 14 Mar 2021) 
389

390

391

392

393

394

395

396

397

398

399

400

401

402

403

404

405

406

407

408

409

410

411

412

413

414

415

416

417

418

419

420

421

Opcode (operation code) is another code-based feature. It is a part of the instruction to inform the CPU regarding the tasks to be fulfilled. Assembly language used this opcode to execute the instruction. Also referred to as bytecode, the examples of an opcode for Android included OP_ADD_DOUBLE, OP_ADD_FLOAT, OP_ADD_INT_2ADDR, and OP_SUB_LONG [88]. Specifically, this feature was adopted in the studies by [89][90] [91] and [92] to detect Android malware in the static analysis. Further examples of the features in this section are method [93], opcode [92], byte stream @ byte block [91], Dalvik code [69], and code involving encryption [94]. The selection of the features by security practitioners is followed by classification. This process was performed to receive the features as input and differentiate between either the application malware or benign (normal).

Figure 6 depicts that researchers prefer to investigate permission and API features compare to others. However, the trend in permission features is decline from 2013 until 2018. However, API features takes place in previous experiments as it increased from six (2014) to 9 (2019). This indicates that the API trend would increase in following year in static detection.

\subsection{Classification}

In the classification process for static analysis, many security analysts used two types of methods; 1) Machine learning (ML) and 2) Graph. The following section presents the ML studies with static features.

\subsubsection{Machine learning (ML)}

Machine learning is a scientific discipline, which is capable to predict future decisions based on the experience it has gained through past inputs (learning set), followed by a prediction of the outputs. Basing on a given dataset, the learning set makes intelligent decisions according to certain algorithms. One of the machine learning types is supervised based on the data for the training stage to create a function. Furthermore, each part of the training data contains input (features or characteristics) and output (class label-malware and benign). This is followed by the training stage, which calculates the approximate distance between the input and output examples to create a model. This training stage could classify unknown applications, such as malware or benign application. Four types of ML are present, such as 1) classical learning; 2) reinforcement learning, 3) neural network and deep learning, and 4) ensemble method. Figure 7 illustrates the ML taxonomy, which starts with classical learning.

a) Supervised Learning

Peer] Comput. Sci. reviewing PDF | (CS-2020:10:54261:2:0:NEW 14 Mar 2021) 
422 Supervised learning (SL) is a process of learning from previous instances to predict future classes. 423 Therefore, the prediction of the class label involves the construction of a concise model from 424 previous experience. The machine learning classifier is then used to test the unknown class [95]. 425 To detect Android malware with static features, the SL method is widely used by security 426 practitioners. Accordingly, the previous articles adopting this method are illustrated in Table 7.

427 b) Unsupervised Learning

428

429

430

431

432

433

434

435

436

437

438

439

440

441

442

443

444

445

446

447

448

449

450

451

452

453

454

Unsupervised learning is another type of learning involved in machine learning. It is a clustering technique where the data is unlabeled and has also been used in computer security areas, including malware detection and forensic [96]. Clustering refers to the division of a large dataset into smaller data sets with several similarities. It classifies a given object set through a certain number of clusters (assume $k$ clusters) to determine the $k$ centroids assigned for each cluster. In this case, this algorithm selects the centroid by random from the applications set, extracts each application from a given dataset, and assigns it to the nearest centroid. Table 7 tabulates the previous articles, which adopted this method.

\section{c) Reinforcement learning}

A reinforcement learning model consists of an agent (a set of actions A) and an environment (the state space S) [97]. Deep reinforcement learning was introduced by reinforcement agents as a framework to play Atari games, which often exceed human performance [98][99]. The advances in deep learning may extract high-level features from raw sensory data, leading to breakthroughs in computer vision and speech recognition. In the case of deep learning, the agent would be required to learn a value function in an end-to-end way, which takes raw pixels as input and predicts the output rewards for each action.

The learned value function is called deep Q learning, in which Q function is learned and refined from over hundreds of games [100]. The Q-learning algorithm was trained in network [98] with stochastic gradient descent to update the weights. Replay mechanism was used from random samples previous transitions to lead smooth training distribution over past behaviors to overcome the correlated data and non-stationary distributions problems. [97] propose a framework based on reinforcement learning (RL) for attacking static portable executable (PE) anti-malware engines. Meanwhile, a DQN-based mobile proposed by [101] to enhance the malware detection performance. The results shown from simulation can increase the malware detection accuracy and reduce the detection delay as compared to a Q-learning based malware detection scheme.

d) Neural Network and Deep Learning 
455 The evolution of Neural Network $(\mathrm{NN})$ has been associated with various challenges since the mid-

456

457

458

459

460

461

462

463

464

465

466

467

468

469

470

471

472

473

474

475

476

477

478

479

480

481

482

483

484

485

486

487

488

489

490

491

492

20th century. McCulloch and Pitts obtained the first inspiration of NN in 1943 from biological neurons, which was followed by proposing a computational model for the development of hypothetical nets. Although this proposal was simulated by Nathaniel Rochester at IBM research laboratory, this attempt was unsuccessful at the end. Developed by Frank Rosenblatt at Cornell Aeronautical Laboratory, the perceptron became the first learning machine [102].

Despite all the upgrades on NNs, Deep learning (DL) was developed in 2006 and has been used in almost every application. As a new variation of the classical Multilayer Perceptron (MLP), the DL aims to produce high-level and flexible features from the raw pixel data to assist in generalising the classification. Furthermore, DL also operates with complex applications containing millions of data, which require a large number of neurons and hidden layers. A few DL frameworks have been developed in the recent years, such as TensorFlow [103], Caffe [104], and Theano [105] to ensure an efficient implementation of Deep Network (DN) architectures and omit the unnecessary coding scratching [102]. Additionally, the DL method extracts the features based on the layer's level, which could either be high or low.

Figure 8 depicts the differences between ML and DL. It shows that ML requires the security practitioners to extract the features manually and select the ML classifier, which is suitable for the selected features. However, DL involves automatic feature extraction part and malware classification. It trains the model end-to-end with the Android application package (.apk) file and their categories, each labelled as malware or benign. The DL gains and creates a prediction model through the automatic selection of the feature.

As one of the major models in deep learning, a convolutional neural network (CNN) has been widely used for image recognition [106]. It could be seen in the past few years that many studies have implemented Deep Neural Networks (DNN) to classify malware [107][108][92]. Additionally, although the recurrent neural networks have been explored since the 1980s, they have become uncommercial due to several issues [107]. Several machine learning methods have addressed network or malware attacks on personal computers or mobile devices. Simultaneously, several techniques were proposed by researchers who applied DL algorithms to detect or categorize malware using static, dynamic, or hybrid approaches, detection of network intrusions and phishing/spam attacks, and inspection of website defacements [109].

\section{e) Ensemble method}

Another technique in machine learning and pattern recognition is ensemble learning. The increase in the implementation of ensemble learning methods could be seen in the computational biology field due to the unique advantages in managing small sample size, high dimension, and complex data structures [110]. The function of ensemble learning is to build a prediction model by

Peer) Comput. Sci. reviewing PDF | (CS-2020:10:54261:2:0:NEW 14 Mar 2021) 
493 combining the strengths of the collection of simpler base models [111]. A few approaches are 494 applied in ensemble methods, such as bagging, boosting, and random forest. This method is also 495 a simple device, which is popular especially in the predictive performance of a base procedure.

496

497

498

499

500

501

502

503

504

505

506

507

508

509

510

511

512

513

514

515

516

517

518

519

520

521

522

523

524

525

526

527

528

529

530

The bagging procedure appears to be a variance reduction scheme for some base procedure, while the boosting methods mainly reduce the bias of the base procedure. Therefore, the significant difference between bagging and boosting ensemble methods is indicated. Compared to bagging and boosting, the random forest approach is a highly distinguished ensemble method. The first proposal of the random forest was made by Amit and Geman [112]. While the performance of random forests is on the same level as boosting, it could exhibit better performance in the perspective of prediction.

Table 8 shows previous works done using different types of machine learnings as mentioned before. From the table, we can summarize classical learning is still valid to be used in experiment execution but there are a lot of works are using deep learning and graph method. The current trends show the demand using the deep learning technique to defend against an increasing number of sophisticated malware attacks where deep learning based have become a vital component of our economic and national security. Many recent studies on Android malware detection have leveraged graph analysis as mentioned in the next section.

\subsubsection{Graph}

The use of a graph is another method in machine learning and pattern recognition, which is performed by investigating the data and control-flow analysis. It is also capable of identifying unknown malware through the examination on the flow of the code. This method is preferred by security analysts due to the uniform flow despite the changes made by the malware authors on the API calls to avoid intrusion detection systems. The types of analysis in graph method include call graph, inter-component call graph (ICCG), control-flow graph (CFG), and dependence graph, while Table 9 lists the previous works of research on static malware detection using the graph method.

A call graph (specifically known as flow graph) is a graph representing the control and data flow of the application, which investigates the exchange of information through the procedures. A node in the graph represents a procedure or function, as seen from the $\mathrm{x}$ and $\mathrm{y}$ symbols, which indicate that procedure $\mathrm{x}$ calls for procedure $\mathrm{y}$. Apposcopy [52] presents its new form of call graph known as inter-component call graph (ICCG) to match malware signature. As a directed graph where nodes are known as components in an application, it establishes ICCG from a call graph and the results of the pointer analysis. The objective of apposcopy is to measure the inter-component communication (ICC), calls, and flow relations. 
531

532

533

534

535

536

537

538

539

540

541

542

543

544

545

546

547

548

549

550

551

552

553

554

555

556

557

558

559

560

561

562

563

564

565

566

567

568
Another graph called a control flow graph (CFG) is also applied by many security analysts to investigate the malware programme. Woodpecker [49] created the CFG start from each entry point (activity, service, receiver, content provider), which is defined in the permission stated in the androidmanifest.xml file. Furthermore, the public interface or services from an execution path is discovered through the flow graph. However, it would be considered by Woodpecker as a capability leak if it is not guarded by the permission requirement nor prevented from being invoked by another unrelated application. The same graph was applied in subsequent works of research, namely Flowdroid [54], Dendroid [86], [76], Asdroid [53], Anadroid [50], Adrisk [44], and Dexteroid [82].

Another graph is the dependency graph, which illustrates the dependencies of several objects on each other. An example could be seen in the dead code elimination case process, in which the graph identifies the dependencies between operation and variables. With the dependency of nonoperation on certain variables, these variables would be considered dead and should be deleted. The studies, which adopted this type of graph are CHEX [113], Dnadroid [114], Droidlegacy [45], and [51].

\subsubsection{Others}

Besides machine learning and graph, several security practitioners adopted different methods, such as Normalized Compression Distance (NCD). Adopted in the studies by [115] and [116], this method can measure the similarities between the malwares and represent them in the form of a distance matrix. Despite the evolution of many malwares from time to time, some of their behaviour patterns are similar to each other. The calculation of the similarities using NCD would identify the malwares, which share the same distance.

A study known as DelDroid [117] implemented a method called as Multiple-Domain Matrix (MDM). This method refers to a complex system, which calculates multiple domains and is based on the Design-Structure Matrix (DSM) model. Furthermore, MDM is formed by the connection of DSM models with each other. The study initialised multiple domains in the MDM to represent the architecture of an Android system for privilege analysis. To illustrate, the incorporation of certain definitions in the MDM representation in the architecture enables DelDroid to identify the communication of the application, which may result in an unauthorised malware attack.

Another previous static experiment was conducted on the MD5 signature of the application to detect malware [58]. In the first process, the study assigned the application as level $\mathrm{C}$ (the lowest level of suspicion), followed by calculation and cross-reference in the database of signatures. The application would be recorded if the result was positive. However, it would be identified as

PeerJ Comput. Sci. reviewing PDF | (CS-2020:10:54261:2:0:NEW 14 Mar 2021) 
569

570

571

572

573

574

575

576

577

578

579

580

581

582

583

584

585

586

587

588

589

590

591

592

593

594

595

596

597

598

599

600

601

602

603

604

605

606

malware if the result of the suspicion was $\mathrm{R}$. The system examined the files inside the application to find any matched MD5 signature.

Androsimilar [91] practised a method known as a statistical similarity digest hashing scheme, which inspects the similarity on the byte stream based on robust statistical malicious static features. It is also a foot-printing method, which identifies the regions or areas of statistical similarity with known malware. Following that, it generates variable-length signatures to detect unknown malware (zero-day).

The following study is DroidMOSS [62], which identifies between the repackaged (modified) and original application. This function is important due to the content of malicious activities in many Android repackaged applications. This study used a fuzzy hashing technique, which generated fingerprint based on this technique to localise and detect any previously applied modifications to the original application. It then calculated the edited distance to measure the similarity between the applications. When the result of the similarity exceeds certain values, the application would be considered as a modified sample.

Under another static experiment, a study by [84] adopted a method known as a risk score weight, which was performed through the calculation of the risk score based on the selected features in the code. When the features were identified, the score increased according to certain risky patterns of properties. Particularly, the patterns were based on different likelihoods of the given situations between normal and malware samples. Lastly, the percentage of the likelihood was calculated. Figure 9 shows that both ML and graph were the popular methods among security practitioners in static analysis. The graph method was found to exceed the ML method in 2011, 2012, and 2014, although ML was more preferred compared to graph in other years. However, this situation reveals that graphs and ML are favourable options in the static experiment.

A study started to utilise DL (part of ML) in the static experiment in 2019, which also combined DL (Convolutional neural network - CNN) with Control flow graph (CFG). Notably, provided that API was the only feature utilised in this study, many future opportunities were available to combine different DL classifiers (Recurrent neural network - RNN, Generative* adversarial networks GAN or Deep belief network* - DBN) with other features besides API and different types of graph. It is noteworthy that DL could also be combined with NCD and MDM.

\section{Open research issues}

This section explains the issues involved in the static analysis, which were derived from previous research articles. Specifically, a discussion is made on the advantages and disadvantages of the open-source operating system, which rely on the availability of the kernel application code.

Peer] Comput. Sci. reviewing PDF | (CS-2020:10:54261:2:0:NEW 14 Mar 2021) 
607 Another issue on static analysis is code obfuscation used by the malware developer to increase the 608 difficulty to detect the malware and research done to counter obfuscation. Then, we review overall 609 static analysis articles, how to detect unknown malware, the combination of static and dynamic, 610 resource consumption, future work, and features.

\section{3.1. Open source advantages and disadvantages}

612 Provided that Android malware is an open-source operating system, there is a continuous increase 613 in its amount. To illustrate, one of the open-source availabilities is the kernel application code. 614 Accordingly, Samsung officially provides its kernel operating system to the public [118] for kernel 615 enhancement or private purposes. Furthermore, any person may download the link according to 616 the mobile device version. The code is also available in Lenovo [119], LG [120], Sony [121], Htc 617 [122], Asus [123], Motorola [124], and other mobile providers. Consequently, this code 618 availability may allow malware writers to identify and manage the vulnerabilities of the kernel's 619 operating system.

620

621 Even though the availability of open-source contributes to its easy access, it is also available for

622

623

624

625

626

627

628

629

630

631

632

633

634

635

636

637

638

639

640

641 security practitioners to research it. These practitioners may be the researchers of universities, the staff of the mobile providers, Android Google researchers, freelance programmers, and the Android community. They also invent various frameworks, algorithms, and suggestions to improve the security of the operating system. The version of the kernel is updated every year, while the mobile providers are informed to regarding the kernel updates. These studies, including static analysis, would increase the confidence of Android users worldwide.

\subsection{Obfuscation}

Static analysis involves reverse engineering, such as decompile and disassemble, while malware developer utilises the obfuscation method to increase the difficulty of the decompiling process and lead to confusion in it. Obfuscation is a technique, which increases the difficulty in understanding the programmes due to the failure of the lead security analysts to distinguish between malware and benign application. Notably, it is a well-known obstacle to be examined by static analysis. Figure 10 illustrates the types of obfuscation, which include encryption, oligomorphic, polymorphism, and metamorphism [125][126].

The encryption method was extensively practised by the malware writers. In this case, the important code or strings, which revealed the malware detector or security practitioner, should be identified. Accordingly, the code was encrypted and converted to the ciphertext. Furthermtore, various algorithms to encrypt the code are present, such as Caesar, Playfair, Data Encryption Standard (DES), Advanced Encryption Standard (AES), and Rivest-Shamir-Adelman (RSA). 
642 Therefore, for the security practitioner to understand the behaviour of the malware, the encrypted

643 code should be decrypted using the correct decryptor [26].

644

645 Besides being a malware capable of mutating @ changing the decryptor, the oligomorphic is also 646 able to generate multiple decryptors to hundreds of types [126]. Consequently, the security 647 practitioner would need to change different decryptor multiple times until the code is returned to 648 the normal string. Nevertheless, this type of obfuscation does not affect the size or shape of the 649 code. Another type of obfuscation is polymorphic. It is a descriptor, which affects the size or shape 650 of the code. Compared to oligomorphic, it is more advanced due to the incorporation of code 651 transposition, register reassignment, dead code@ nop insertion, and armoring. Meanwhile, 652 metamorphism is an approach beyond the oligomorphic and polymorphic types due to the absence 653 of decryptor in its mechanism. Therefore, its constant body could be hidden from memory and 654 655

656 The following information is the obfuscation methods that regularly used by polymorphism 657 (polimorphic) and metamorphism (metamorphic) obfuscation [126].

658 a) Code transportation

659 Code transposition is a method, which restructures the orders of the original code without causing 660 any effects on its conduct. This process is performed with two methods. The first method is the 661 random restructure of the original code by including jumps or unconditional branches. However, 662 security practitioners can detect obfuscation by removing those jumps or unconditional branches. 663 The second method is the production of new generations by selecting and restructuring 664 independent instructions without any impact on others. However, the adoption of these methods is 665 challenging for the malware writer, while the security practitioners are faced with a difficulty to 666 detect this method of obfuscation.

667 b) Register reassignment

668 Register reassignment is another method of obfuscation, which shifts the registers of the code from 669 one generation to another. This method is performed without changing the behaviour of the code 670 while keeping the programme of the code similar to its original state.

671 c) Dead-code/nop insertion

672 Known as nop insertion, dead-code is a method, which adds several unnecessary instructions in 673 the code and simultaneously keeps the behaviour of the code similar to its original state. 674 Nevertheless, in certain situations, security practitioners able to detect this obfuscation by 675 removing the aforementioned code. 
676 d) Substitution of equivalent instruction sequences

677 The original code is changed through the substitution of several instructions. To illustrate, the SUB 678 instruction is changed to XOR, while PUSH is changed to MOV.

679

680

\subsubsection{Research to counter obfuscation}

681 To overcome obfuscation, many studies were conducted on different approaches. Study by [114] 682 used program dependence graph (PDG) to prevent program transformations in obfuscation. 683 Droidlegacy [45] use graph node to represents the java class in detecting light obfuscation. 684 Droidanalytics [89] and Drebin [55] extract the API calls while the codes running during execution 685 time. In order to control the flow of obfuscation, Apposcopy use inter-component communication 686 (ICC) to write the signature. Research by [127] uses jadx, one of reverse engineer tool to de687 obfuscation the obfuscation codes. Summary of studies conducted to overcome obfuscation shown 688 in Table 10.

\section{$689 \quad 3.2 .2 \quad$ Advantage of obfuscation}

690 Despite the adoption of the obfuscation method by the malware writers or the attackers to evade 691 detection, obfuscation also serves the following advantages based on other points of views:

692 a) Reduction of the size of the application

693 Google [128] encourages developers to enable shrinking in their release to build an application to 694 remove any unused codes and resources. Furthermore, provided that obfuscation would shorten 695 the names of the classes and members in the code, the developer will be able to reduce the size of 696 the application. Notably, the size of the application is a significant concern in Android handheld 697 devices (smartphones, smart glasses, and smartwatch) with limited storage and resources.

698 b) The difficulty for the malware writer to understand the obfuscated normal application

699 To develop malware in certain situations, malware writers need to perform reverse engineering on 700 the normal repackaged application. Therefore it is able to confuse them to steal private information 701 and discover application vulnerabilities from that obfuscated normal @ benign application code 702 [129]. 
703

704

705

706

707

708

709

710

711

712

713

714

715

716

717

718

719

720

721

722

723

724

725

726

727

728

729

730

731

732

733

734

735

736

737

c) Security practitioners can detect malware easily

Obfuscation also facilitates the detection of malware by the researcher [130]. To illustrate, there are certain situations where malware regularly adopts similar obfuscation marks, which is impossible to exist in normal application. Therefore, security practitioners able to detect malware with the presence of these marks. Following all the advantages and drawbacks, continuous research on obfuscation is crucial to obtain better results from the detection of malware through the static analysis.

\subsection{The list of all articles in the detection of malware in static analysis}

To identify the trends in the detection of malware through static analysis, this section presents a list of previous works of research, which cover all areas (year, features, and classification). Table 11 lists a study DroidARA [131] in 2019, which performed an experiment combined with DL and graph and differentiation between malware and normal application. It applied a call graph to extract the API features and convolutional neural network (CNN) for classification. At the time of writing this paper, this is a new trend in detecting Android malware. Therefore, in future research, it is possible to witness more research combination similar to this with different features.

From the lists, most of researchers used API and manifest file features in their experiments to detect the malware. It proofs that API features were the popular codes used by the malware developers to create the malware. The program is based on the Android application package file in the (.apk) format, which is also used to install an application in android-based mobile devices. Every app project must have an androidmanifest.xml file at the root of the project source set. This manifest file is regularly in a binary form inside the APK, however when chosen in the APK Analyzer, the $\mathrm{xml}$ form is restructured and produced. There are some changes of the androidmanifest.xml document from a library application depends on was converged into the last androidmanifest.xml record. Other package files fall down into apk, xml and dex properties feature.

Besides the combination of DL and graph, ML and graph were also combined in the studies by [87] in 2016 and [76] in 2012. These studies utilised a similar graph, which was the Control flow graph (CFG), indicating that the combination of ML and graph increased the detection result. Therefore, future work is suggested to test this combination in different static features. Other parts of classification (Multiple-Domain Matrix (MDM), MD5 signature, similarity digest hashing, normalized compression distance (NCD), and fuzzy hashing technique) were also useful in the detection of malware with static features. These classifications also contributed to the availability of future work combinations with ML, DL, and graph. 
738

739

740

741

742

743

744

745

746

747

748

749

750

751

752

753

754

755

756

757

758

759

760

761

762

763

764

765

766

767

768

769

770

771

772

\subsection{Detect unknown malware}

Initially, static analysis is unable to discover new or unknown malware as it only examined the code of the application without executing it. To elaborate on this situation, certain malware only executes certain parts whenever the application runs. Provided the drawback of static analysis in the identification of unknown malware, many security practitioners started to adopt machine learning, such as [132][13], Drebin [55], [57], Droidapiminer [30], [133], Androsimilar [91], [46], [134], [116], [64], and [65]. Similarly, the graph approach was also a suitable approach for this identification, as shown in [135], Riskranker [136], and Dendroid [86]. The [135] study utilised a data dependence graph (DDG), Riskranker, and Dendroid, which employed the control-flow graph (CFG).

\subsection{Combination of static and dynamic analyses}

It was proven in [125] that static analysis was inadequate for the detection of malware as this analysis should be combined with dynamic analysis to detect the malware effectively. Compared to static analysis, the dynamic analysis can evade the obfuscation technique. Essentially, provided that each type of analysis (static and dynamic) has its advantages and drawbacks, the combination of static and dynamic analyses would increase the effectiveness of the countermeasure action on the malware.

\subsection{Resource consumption in Android OS and deep learning}

Deep learning (DL) is a subset of machine learning in artificial intelligence (AI), which is also known as a deep neural network or deep neural learning. Notably, with unlabeled and unstructured data, DL is capable of learning and predicting the output. It imitates the human brain in data processing, development of patterns from that data, and the implementation of decision making. It could be seen from the current trends that deep learning (DL) technique has a potential for further discovery. The implementation of this technique enables the DL to automatically determine the ideal features for prediction and classification. Currently, DL is widely used in almost every area, such as large scale image recognition tasks, automated driving, new cancer cell detection, hearing and speech translation, and aerospace area identification [137].

However, DL requires substantial computing power, which needs a high amount of the graphic processing unit (GPU) based on the data to be processed [137]. This situation leads to an issue in the detection of malware, which attacks Android devices. Provided that Android mobile device is a device with small computing power, the adoption of DL becomes the main concern. However, the transfer of information from the Android device to the cloud provider is possible only for the execution of the DL process, which would then develop the device. Therefore, the large-scale adoption of DL is possible for future work in the static analysis. 


\section{3.7. Future work in static analysis}

774 It could be seen from the review in the previous sections (section 2) that many future opportunities

775 for the static analysis to detect the malware, which attacks the Android. One of the opportunities

776 is the combination of different DL classifier (Recurrent neural network - RNN, Generative*

777 adversarial networks - GAN or Deep belief network* - DBN) with other features besides API,

778 with different types of graph. However, Section 3.3 shows that only one experiment started the 779 combination between DL and graph with one API feature in 2019. Therefore, the accuracy of

780 detection results would increase, leading to the identification of a new family of malware. It is also 781 noteworthy that other future alternatives are available for the combination of DL with NCD and 782 MDM.

\section{3.8. Popular features (API and manifest file)}

784 Section 3.3 shows that many static analysis researchers frequently applied the manifest file and 785 API calls as the features in their experiments. To illustrate, these popular features had been 786 examined by the researchers from 2010 until 2019 due to the official update for Android and the 787 addition of new features from time to time. However, most of the malwares still utilised similar 788 features within this timespan, while other malwares utilised the latest and updated features.

789

790

791

792

793

794

795

796

797

798

799

800

801

802

803

804

805

806

807

808

809 Therefore, the researchers are required to place continuous focus on these popular features.

The manifest file is one file with numerous features in it, such as permission, intent, hardware component, and application component, while API is a ready code for the programmer to develop their application. Therefore, it is crucial for researchers to thoroughly scrutinise these two categories of features. Moreover, some researchers incorporated other features to combine the manifest and API for more effective malware detection.

\section{Conclusions}

Following the interest to explore the recent studies in the static analysis, a review was performed on the existing studies by past security investigators on Android malware detection, which was explained through phases (reverse engineer, features, and classification). Furthermore, this review covered the information within the ten years range (2009 to 2019). In this article, the features used in the static analysis were also reviewed. Within the aforementioned timespan, many security practitioners still preferred the API and manifest files, indicating the relevance of these two features. Moreover, the latest trends in classification were highlighted, which consists of machine learning, deep learning, graph, and other methods. These trends have proven the relevance of the graph method compared to machine learning. Static analysis researchers began the adoption of deep learning in their detection. This article also discussed the open research issues in the static analysis, including obfuscation as one of the weaknesses of static analysis and the methods of 
810 overcoming it. Many static analysis researchers implemented multiple methods to solve 811 obfuscation and achieve a successful malware detection, such as concentrating the codes with the 812 implementation of obfuscation (DexClassLoader.loadClass, Crypto.spec.DESKeySpec, and 813 Cipher.getInstance) using a tool with the de-obfuscation option, including the adoption of graph 814 node and program dependence graphs (PDGs).

815

816 Acknowledgements

817

818 This study thanked to Ministry of Higher Education (MOHE) for Fundamental Research Grant 819 Scheme (FRGS) with grant number RDU190190, FRGS/1/2018/ICT02/UMP/02/13, and 820 Universiti Malaysia Pahang (UMP) internal grant with grant number RDU180337.

821

822

\section{References}

823

824

825

826

827

828

829

830

831

832

833

834

835

836

837

838

839

840

841

842

843

844

845

846

847

848

849

850

851

852

853

[1] T. Rayner, “Android Power Rankings (May 2019): Shake-up at the top,” 2019. https://www.androidauthority.com/android-power-rankings-may-2019-980770/ (accessed Sep. 30, 2019).

[2] Jkielty, “Android v iOS market share 2019." https://deviceatlas.com/blog/android-v-iosmarket-share (accessed Sep. 30, 2019).

[3] Android, “Google," 2019. https://www.android.com/what-is-android/ (accessed Sep. 30, 2019).

[4] Statista, "Development of new Android malware worldwide from January 2011 to March 2018," 2019. https://www.statista.com/statistics/680705/global-android-malware-volume/ (accessed Sep. 30, 2019).

[5] McAfee, "Mobile Threat Report," 2019. https://www.mcafee.com/enterprise/en-us/threatcenter/mcafee-labs/reports.html-mobile-threat-report-2018.pdf.

[6] V. Beal, "Mobile Malware." https://www.webopedia.com/TERM/M/mobile_malware.html.

[7] Y. Ma and M. S. Sharbaf, "Investigation of Static and Dynamic Android Anti-virus Strategies," in 10th International Conference on Information Technology: New Generations (ITNG), Las Vegas, Nevada, Apr. 2013, pp. 398-403, doi: 10.1109/ITNG.2013.62.

[8] S. A. Aubrey-Derrick Schmidt, Hans-Gunther Schmidt, Leonid Batyuk, Jan Hendrik Clausen, Seyit Ahmet Camtepe, "Smartphone malware evolution revisited: Android next target?," 2009 4th Int. Conf. Malicious Unwanted Software, MALWARE 2009, no. November 2018, pp. 1-7, 2009, doi: 10.1109/MALWARE.2009.5403026.

[9] L. O’Donnell, “Google Play Malicious Apps Racked Up 335M+ Installs in September," 2019. https://threatpost.com/google-play-malicious-apps-racked-up-335m-installs-inseptember/148810/ (accessed Nov. 08, 2019).

[10] W. Enck, "Defending Users against Smartphone Apps: Techniques and Future Directions," in Proceedings of the 7th international conference on Information Systems Security, Kolkata, India, 2011, pp. 49-70.

[11] I. Yaqoob, I. Abaker, T. Hashem, A. Ahmed, and S. M. A. Kazmi, "Internet of things forensics: Recent advances, taxonomy, requirements, and open challenges," Futur. Gener. 
854

855

856

857

858

859

860

861

862

863

864

865

866

867

868

869

870

871

872

873

874

875

876

877

878

879

880

881

882

883

884

885

886

887

888

889

890

891

892

893

894

895

896

897

898

899

Comput. Syst., vol. 92, no. May 2018, pp. 265-275, 2019, doi: S0167739X18315644.

[12] C. A. Castillo, "Android Malware Past, Present, and Future," McAfee White Paper, Mobile Security Working Group, 2011. .

[13] S. Y. Yerima, S. Sezer, and G. McWilliams, "Analysis of Bayesian classification-based approaches for Android malware detection," IET Inf. Secur., vol. 8, no. 1, pp. 25-36, 2014, doi: 10.1049/iet-ifs.2013.0095.

[14] H. Y. Saba Arshad, Munam A Shah, Abdul Wahid, Amjad Mehmood, Houbing Song, "SAMADroid: A Novel 3-Level Hybrid Malware Detection Model for Android Operating System," IEEE Access, vol. 6, pp. 4321-4339, 2018, doi:

10.1109/ACCESS.2018.2792941.

[15] M. Spreitzenbarth, T. Schreck, F. Echtler, D. Arp, and J. Hoffmann, "Mobile-Sandbox : combining static and dynamic analysis with machine-learning techniques," pp. 141-153, 2015, doi: 10.1007/s10207-014-0250-0.

[16] Y. Fang, Y. Gao, F. A. N. Jing, and L. E. I. Zhang, "Android Malware Familial Classification Based on DEX File Section Features," vol. 8, 2020.

[17] F. Alswaina and K. Elleithy, "Android malware family classification and analysis: Current status and future directions," Electron., vol. 9, no. 6, pp. 1-20, 2020, doi: 10.3390/electronics9060942.

[18] A. Feizollah, N. B. Anuar, R. Salleh, and A. W. A. Wahab, "A review on feature selection in mobile malware detection," Digit. Investig., vol. 13, pp. 22-37, 2015, doi: 10.1016/j.diin.2015.02.001.

[19] Sufatrio, D. J. J. Tan, T. Chua, and V. L. L. Thing, "Securing Android: A Survey, Taxonomy, and Challenges," ACM Comput. Surv., vol. 47, no. 4, pp. 1-45, 2015.

[20] S. Schmeelk, J. Yang, and A. Aho, "Android Malware Static Analysis Techniques," in CISR '15 Proceedings of the 10th Annual Cyber and Information Security Research Conference, 2015, pp. 1-8.

[21] Z. Fang, W. Han, and Y. Li, "Permission based Android security: Issues and countermeasures," Comput. Secur., vol. 43, no. 0, pp. 205-218, 2014, doi: 10.1016/j.cose.2014.02.007.

[22] Y. Pan, X. Ge, C. Fang, and Y. Fan, "A Systematic Literature Review of Android Malware Detection Using Static Analysis," IEEE Access, pp. 1-18, 2020, doi: 10.1109/ACCESS.2020.3002842.

[23] H. A. M. Shaffril, S. E. Krauss, and S. F. Samsuddin, "A systematic review on Asian's farmers' adaptation practices towards climate change," Sci. Total Environ., vol. 644, pp. 683-695, 2018, doi: 10.1016/j.scitotenv.2018.06.349.

[24] A. Feizollah, N. B. Anuar, R. Salleh, F. Amalina, R. R. Ma'arof, and S. Shamshirband, "A Study Of Machine Learning Classifiers For Anomaly-Based Mobile Botnet Detection," Malaysian J. Comput. Sci., vol. 26, no. 4, pp. 251-265, 2013.

[25] S. Y. Yerima, S. Sezer, and I. Muttik, "High Accuracy Android Malware Detection Using Ensemble Learning," IET Inf. Secur., vol. 9, no. 6, pp. 313-320, 2015, doi: 10.1049/ietifs.2014.0099.

[26] F. Wei, Y. Li, S. Roy, X. Ou, and W. Zhou, "Deep ground truth analysis of current android malware," Lect. Notes Comput. Sci. (including Subser. Lect. Notes Artif. Intell. Lect. Notes Bioinformatics), vol. 10327 LNCS, pp. 252-276, 2017, doi: 10.1007/978-3319-60876-1_12.

[27] B. Chess and G. McGraw, "Static analysis for security," IEEE Security \& Privacy 
900

901

902

903

904

905

906

907

908

909

910

911

912

913

914

915

916

917

918

919

920

921

922

923

924

925

926

927

928

929

930

931

932

933

934

935

936

937

938

939

940

941

942

943

944

945

Magazine, vol. 2, no. 6, pp. 76-79, 2004.

[28] M. Sharif, V. Yegneswaran, H. Saidi, P. Porras, and W. Lee, "Eureka: A framework for enabling static malware analysis," in Lecture Notes in Computer Science, vol. 5283, 2008, pp. 481-500.

[29] T.-K. Chang and G.-H. Hwang, "The design and implementation of an application program interface for securing XML documents," J. Syst. Softw., vol. 80, no. 8, pp. 13621374, 2007, doi: 10.1016/j.jss.2006.10.051.

[30] Y. Aafer, W. Du, and H. Yin, "DroidAPIMiner: Mining API-Level Features for Robust Malware Detection in Android," in Security and Privacy in Communication Networks, 2013, pp. 86-103.

[31] F. A. Narudin, A. Feizollah, N. B. Anuar, and A. Gani, "Evaluation of machine learning classifiers for mobile malware detection," Soft Comput., vol. 20, no. 1, pp. 343-357, Nov. 2014, doi: 10.1007/s00500-014-1511-6.

[32] A. A. A. Samra, H. N. Qunoo, F. Al-Rubaie, and H. El-Talli, "A survey of Static Android Malware Detection Techniques," 2019 IEEE 7th Palest. Int. Conf. Electr. Comput. Eng., pp. 1-6, 2019, doi: 10.1109/picece.2019.8747224.

[33] D. Gibert, C. Mateu, and J. Planes, "The rise of machine learning for detection and classification of malware: Research developments, trends and challenges," J. Netw. Comput. Appl., vol. 153, no. November 2019, p. 102526, 2020, doi: 10.1016/j.jnca.2019.102526.

[34] "Malgenome Project." http://www.malgenomeproject.org/.

[35] K. Allix, T. F. Bissyandé, J. Klein, and Y. Le Traon, "AndroZoo: Collecting Millions of Android Apps for the Research Community," in MSR '16 Proceedings of the 13th International Conference on Mining Software Repositories, Austin, Texas, 2016, pp. 468471, doi: 10.1145/2901739.2903508.

[36] U. du Luxembourg, "Androzoo," 2016. https://androzoo.uni.lu/.

[37] “Drebin dataset." http://www.sec.cs.tu-bs.de/ danarp/drebin/.

[38] “Android Malware Dataset," 2019. .

[39] “AMD dataset." http://amd.arguslab.org/.

[40] "Contagio Mobile," 2019. .

[41] R. Dhaya and M. Poongodi, "Detecting software vulnerabilities in android using static analysis," Proc. 2014 IEEE Int. Conf. Adv. Commun. Control Comput. Technol.

ICACCCT 2014, no. 978, pp. 915-918, 2015, doi: 10.1109/ICACCCT.2014.7019227.

[42] M. Chanda and M. Biswas, "Plant Diseases Classification Using Feature Reduction, BPNN And PSO," Int. J. Softw. Eng. Comput. Syst., vol. 5, no. 2, pp. 90-103, 2019.

[43] M. F. J. Klaib, M. R. A. Sara, and M. Hasan, "D-GREEDY: Greedy shortest superstring with delayed random choice," Int. J. Softw. Eng. Comput. Syst., vol. 6, no. 1, pp. 8-17, 2020.

[44] M. C. Grace, W. Zhou, X. Jiang, and A.-R. Sadeghi, "Unsafe Exposure Analysis of Mobile In-App Advertisements," in Proceeding 5th ACM conference on Security and Privacy in Wireless and Mobile Networks, Tucson, Arizona, USA, 2012, vol. 67, no. 2, pp. 101-112, doi: 10.1145/2185448.2185464.

[45] L. Deshotels, V. Notani, and A. Lakhotia, "DroidLegacy: Automated Familial Classification of Android Malware," in Proceedings of ACM SIGPLAN on Program Protection and Reverse Engineering Workshop, San Diego, CA, USA, 2014, pp. 1-12, doi: $10.1145 / 2556464.2556467$. 
946 [46] S.-H. Lee and S.-H. Jin, "Warning System for Detecting Malicious Applications on

947

948

949

950

951

952

953

954

955

956

957

958

959

960

961

962

963

964

965

966

967

968

969

970

971

972

973

974

975

976

977

978

979

980

981

982

983

984

985

986

987

988

989

990

991
Android System," Int. J. Comput. Commun. Eng., vol. 2, no. 3, pp. 324-327, 2013, doi:

10.7763/IJCCE.2013.V2.197.

[47] D.-J. Wu, C.-H. Mao, T.-E. Wei, H.-M. Lee, and K.-P. Wu, "DroidMat: Android Malware Detection through Manifest and API Calls Tracing," in Seventh Asia Joint Conference on Information Security, Tokyo, Japan, Aug. 2012, pp. 62-69, doi:

10.1109/AsiaJCIS.2012.18.

[48] A. Bartel, J. Klein, Y. Le Traon, and M. Monperrus, "Automatically securing permissionbased software by reducing the attack surface: an application to Android," in Proceedings of the 27th IEEE/ACM International Conference on Automated Software Engineering (ASE), Essen, Germany, 2012, pp. 274-277, doi: 10.1145/2351676.2351722.

[49] M. Grace, Y. Zhou, Z. Wang, and X. Jiang, "Systematic Detection of Capability Leaks in Stock Android Smartphones," in Proceedings of the 19th Network and Distributed System Security Symposium (NDSS), San Diego, CA, 2012, pp. 1-15.

[50] D. V. H. Shuying Liang, Andrew W. Keep, Matthew Might, Thomas Gilray, Steven Lyde, Petey Aldous, "Sound and Precise Malware Analysis for Android via Pushdown Reachability and Entry-Point Saturation," in ACM workshop on Security and privacy in smartphones and mobile devices, Berlin, Germany, 2013, pp. 21-32, doi: 10.1145/2516760.2516769.

[51] W. Zhou, Y. Zhou, M. Grace, X. Jiang, and S. Zou, "Fast, scalable detection of 'Piggybacked' mobile applications," in CODASPY'13 Proceedings of the second ACM conference on Data and Application Security and Privacy, San Antonio, Texas, USA, 2013, pp. 185-195, doi: 10.1145/2435349.2435377.

[52] Y. Feng, S. Anand, I. Dillig, and A. Aiken, "Apposcopy: semantics-based detection of Android malware through static analysis," Proc. 22nd ACM SIGSOFT Int. Symp. Found. Softw. Eng. - FSE 2014, pp. 576-587, 2014, doi: 10.1145/2635868.2635869.

[53] J. Huang, X. Zhang, L. Tan, P. Wang, and B. Liang, “AsDroid: Detecting Stealthy Behaviors in Android Applications by User Interface and Program Behavior Contradiction," in Proceeding ICSE 2014 Proceedings of the 36th International Conference on Software Engineering, Hyderabad, India, 2014, pp. 1036-1046, doi: $10.1145 / 2568225.2568301$.

[54] S. R. Steven Arzt, J. K. Christian Fritz, Eric Bodden, Alexandre Bartel, and P. M. and Yves Le Traon, Damien Octeau, "FlowDroid: Precise Context, Flow, Field, Objectsensitive and Lifecycle-aware Taint Analysis for Android Apps," in Proceedings of the 35th ACM SIGPLAN Conference on Programming Language Design and Implementation, Edinburgh, United Kingdom, 2014, pp. 259-269.

[55] D. Arp, M. Spreitzenbarth, H. Malte, H. Gascon, and K. Rieck, "DREBIN: Effective and Explainable Detection of Android Malware in Your Pocket," in 21th Annual Network and Distributed System Security Symposium (NDSS), San Diego, CA, 2014, pp. 1-15.

[56] Z. Luoshi, N. Yan, W. Xiao, Z. Wang, and Y. Xue, “A3: Automatic Analysis of Android Malware," in International Workshop on Cloud Computing and Information Security (CCIS), Shanghai, China, 2013, pp. 89-93, doi: 10.2991/ccis-13.2013.22.

[57] S. Y. Yerima, S. Sezer, and I. Muttik, "Android Malware Detection Using Parallel Machine Learning Classifiers," in Eight International Conference on Next Generation Mobile Apps, Services and Technologies, (NGMAST), St. Anthony's College of the University of Oxford, UK, 2014, pp. 37-42, doi: 10.1109/NGMAST.2014.23. 
992 [58] S.-H. Seo, A. Gupta, A. Mohamed Sallam, E. Bertino, and K. Yim, "Detecting mobile

993

994

995

996

997

998

999

1000

1001

1002

1003

1004

1005

1006

1007

1008

1009

1010

1011

1012

1013

1014

1015

1016

1017

1018

1019

1020

1021

1022

1023

1024

1025

1026

1027

1028

1029

1030

1031

1032

1033

1034

1035

1036

1037 malware threats to homeland security through static analysis," J. Netw. Comput. Appl., vol. 38, pp. 43-53, 2014, doi: 10.1016/j.jnca.2013.05.008.

[59] S. Sheen, R. Anitha, and V. Natarajan, "Android based malware detection using a multifeature collaborative decision fusion approach," Neurocomputing, vol. 151, pp. 905912, 2015, doi: 10.1016/j.neucom.2014.10.004.

[60] N. Peiravian and X. Zhu, "Machine Learning for Android Malware Detection Using Permission and API Calls," in International Conference on Tools with Artificial Intelligence (ICTAI), Herndon, VA, USA, 2013, pp. 300-305, doi:

10.1109/ICTAI.2013.53.

[61] H. Kang, J. Jang, A. Mohaisen, and H. K. Kim, "Detecting and Classifying Android Malware using Static Analysis along with Creator Information," Int. J. Distrib. Sens. Networks - Spec. issue Adv. Big Data Manag. Anal. Ubiquitous Sensors, vol. 11, no. 6, pp. 1-17, 2015, doi: 10.1155/2015/479174.

[62] W. Zhou, Y. Zhou, X. Jiang, and P. Ning, "Detecting Repackaged Smartphone Applications in Third-Party Android Marketplaces," in CODASPY '12 Proceedings of the second ACM conference on Data and Application Security and Privacy, San Antonio, Texas, USA, 2012, pp. 317-326, doi: 10.1145/2133601.2133640.

[63] A. A. A. Samra, Y. Kangbin, and O. A. Ghanem, "Analysis of Clustering Technique in Android Malware Detection," in Seventh International Conference on Innovative Mobile and Internet Services in Ubiquitous Computing (IMIS), Taichung, Taiwan, 2013, pp. 729 733, doi: 10.1109/IMIS.2013.111.

[64] A. Shabtai, Y. Fledel, and Y. Elovici, "Automated Static Code Analysis for Classifying Android Applications Using Machine Learning," in Ninth International Conference on Computational Intelligence and Security, Nanning, Guangxi Zhuang Autonomous Region China, Dec. 2010, pp. 329-333, doi: 10.1109/CIS.2010.77.

[65] A. Firdaus and N. B. Anuar, "Root-exploit Malware Detection using Static Analysis and Machine Learning," in Proceedings of the Fourth International Conference on Computer Science \& Computational Mathematics (ICCSCM 2015), Langkawi, Malaysia, 2015, pp. 177-183.

[66] Android Developer, "Android Debug Bridge (ADB)," 2017. .

[67] A. Firdaus, N. B. Anuar, M. F. A. Razak, I. A. T. Hashem, S. Bachok, and A. K. Sangaiah, "Root Exploit Detection and Features Optimization: Mobile Device and Blockchain Based Medical Data Management," J. Med. Syst., vol. 42, no. 6, 2018, doi: 10.1007/s10916-018-0966-X.

[68] O. K. Aubrey-Derrick Schmidt, Rainer Bye, Hans-Gunther Schmidt, Jan Clausen and and S. A. Kamer A. Yuksel, Seyit A. Camtepe, "Static Analysis of Executables for Collaborative Malware Detection on Android," in IEEE International Conference on Communications (ICC), Dresden, Germany, Jun. 2009, pp. 1-5, doi:

10.1109/ICC.2009.5199486.

[69] H. Gascon, F. Yamaguchi, D. Arp, and K. Rieck, "Structural Detection of Android Malware using Embedded Call Graphs," in Proceedings of the 2013 ACM workshop on Artificial Intelligence and Security, Berlin, Germany, 2013, pp. 45-54, doi: 10.1145/2517312.2517315.

[70] Android, "App Manifest," 2015.

https://developer.android.com/guide/topics/manifest/manifest-intro (accessed Oct. 02, 
1038

1039

1040

1041

1042

1043

1044

1045

1046

1047

1048

1049

1050

1051

1052

1053

1054

1055

1056

1057

1058

1059

1060

1061

1062

1063

1064

1065

1066

1067

1068

1069

1070

1071

1072

1073

1074

1075

1076

1077

1078

1079

1080

1081

1082

1083

2019).

[71] M. F. A. Razak, N. B. Anuar, F. Othman, A. Firdaus, F. Afifi, and R. Salleh, "Bioinspired for Features Optimization and Malware Detection," Arab. J. Sci. Eng., vol. 43, no. 12, pp. 6963-6979, 2018, doi: 10.1007/s13369-017-2951-y.

[72] M. F. A. Razak, N. B. Anuar, R. Salleh, A. Firdaus, M. Faiz, and H. S. Alamri, "“Less Give More': Evaluate and zoning Android applications," Measurement, vol. 133, pp. 396411, 2019, doi: 10.1016/j.measurement.2018.10.034.

[73] C. G. Hao Peng and I. M. Bhaskar Sarma, Ninghui Li, Yuan Qi, Rahul Potharaju, Cristina Nita-Rotaru, "Using Probabilistic Generative Models for Ranking Risks of Android Apps," in ACM Conference on Computer and Communications Security, (CCS), Raleigh, North Carolina, USA, 2012, pp. 241-252, doi: 10.1145/2382196.2382224.

[74] A. Walenstein, L. Deshotels, and A. Lakhotia, "Program Structure-Based Feature Selection for Android Malware Analysis," in Lecture Notes of the Institute for Computer Sciences, Social Informatics and Telecommunications Engineering, vol. 107, 2012, pp. 51-52.

[75] C.-Y. Huang, Y.-T. Tsai, and C.-H. Hsu, "Performance Evaluation on Permission-Based Detection for Android Malware," in Proceedings of the International Computer Symposium ICS 2012 Held at Hualien, Taiwan, 2012, vol. 21, pp. 111-120, doi: 10.1007/978-3-642-35473-1.

[76] J. Sahs and L. Khan, "A Machine Learning Approach to Android Malware Detection," in European Intelligence and Security Informatics Conference, (EISIC), Odense, University of Southern Denmark, 2012, pp. 141-147, doi: 10.1109/EISIC.2012.34.

[77] B. Sanz, I. Santos, C. Laorden, X. Ugarte-Pedrero, P. G. Bringas, and G. Alvarez, "PUMA: Permission Usage to detect Malware in Android," in Advances in Intelligent Systems and Computing, 2013, pp. 289-298.

[78] K. A. Talha, D. I. Alper, and C. Aydin, "APK Auditor: Permission-based Android malware detection system," Digit. Investig., vol. 13, pp. 1-14, 2015, doi: 10.1016/j.diin.2015.01.001.

[79] Z. Aung and W. Zaw, "Permission-Based Android Malware Detection," Int. J. Sci. Technol. Res., vol. 2, no. 3, pp. 228-234, 2013.

[80] A. Feizollah, N. B. Anuar, R. Salleh, G. Suarez-Tangil, and S. Furnell, “AndroDialysis: Analysis of Android Intent Effectiveness in Malware Detection," Comput. Secur., vol. 65, pp. 121-134, 2017, doi: 10.1016/j.cose.2016.11.007.

[81] M. Fazeen and R. Dantu, "Another free app: Does it have the right intentions?," Twelfth Annu. Int. Conf. Privacy, Secur. Trust. Toronto, ON, Canada, pp. 282-289, 2014, doi: 10.1109/PST.2014.6890950.

[82] M. Junaid, D. Liu, and D. Kung, "Dexteroid: Detecting Malicious Behaviors in Android Apps Using Reverse-Engineered Life Cycle Models," Comput. Secur., vol. 59, pp. 92117, 2016, doi: 10.1016/j.cose.2016.01.008.

[83] A. A. Allahham and M. A. Rahman, "a Smart Monitoring System for Campus Using Zigbee Wireless Sensor Networks,” Int. J. Softw. Eng. Comput. Syst., vol. 4, no. 1, pp. 114, 2018, doi: 10.15282/ijsecs.4.1.2018.1.0034.

[84] A. Apvrille and T. Strazzere, "Reducing the window of opportunity for Android malware Gotta catch 'em all,' J. Comput. Virol., vol. 8, no. 1-2, pp. 61-71, 2012, doi:

10.1007/s11416-012-0162-3.

[85] N. O. Mohd Azwan Hamza, Mohd Juzaiddin Ab Aziz, "Sentence Similarity Measurement 
1084

1085

1086

1087

1088

1089

1090

1091

1092

1093

1094

1095

1096

1097

1098

1099

1100

1101

1102

1103

1104

1105

1106

1107

1108

1109

1110

1111

1112

1113

1114

1115

1116

1117

1118

1119

1120

1121

1122

1123

1124

1125

1126

1127

1128

1129

Based on Thematic Role and Semantic Network Techniques," Concept Commun., vol. null, no. 23, pp. 301-316, 2019, doi: 10.15797/concom.2019..23.009.

[86] G. Suarez-Tangil, J. E. Tapiador, P. Peris-Lopez, and J. Blasco, "Dendroid: A text mining approach to analyzing and classifying code structures in Android malware families," Expert Syst. Appl., vol. 41, no. 4 PART 1, pp. 1104-1117, 2014, doi: 10.1016/j.eswa.2013.07.106.

[87] M. A. Atici, S. Sagiroglu, and I. A. Dogru, "Android Malware Analysis Approach Based on Control Flow Graphs and Machine Learning Algorithms," in IEEE 4th International Symposium on Digital IForensics and Security, Little Rock, Arkansas, 2016, pp. 26-31, doi: 10.1109/ISVLSI.2016.35.

[88] A. Developer, "Opcode," 2020. .

[89] M. Zheng, M. Sun, and J. C. S. Lui, "Droid analytics: A signature based analytic system to collect, extract, analyze and associate android malware," in 12th IEEE International Conference on Trust, Security and Privacy in Computing and Communications, (TrustCom), Melbourne, VIC, Australia, 2013, pp. 163-171, doi:

10.1109/TrustCom.2013.25.

[90] E. Medvet and F. Mercaldo, "Exploring the usage of topic modeling for android malware static analysis," 11th Int. Conf. Availability, Reliab. Secur. (ARES), Salzburg, Austria, pp. 609-617, 2016, doi: 10.1109/ARES.2016.10.

[91] P. Faruki, V. Ganmoor, V. Laxmi, M. S. Gaur, and A. Bharmal, "AndroSimilar: Robust Statistical Feature Signature for Android Malware Detection," in Proceedings of the 6th International Conference on Security of Information and Networks, Aksaray, Turkey, 2013, pp. 152-159.

[92] L. Zhao, D. Li, G. Zheng, and W. Shi, "Deep neural network based on android mobile malware detection system using opcode sequences," Int. Conf. Commun. Technol. Proceedings, ICCT, pp. 1141-1147, 2019, doi: 10.1109/ICCT.2018.8600052.

[93] H. Kim, T. Cho, G. J. Ahn, and J. Hyun Yi, "Risk assessment of mobile applications based on machine learned malware dataset," Multimed. Tools Appl., vol. 77, no. 4, pp. 5027-5042, 2018, doi: 10.1007/s11042-017-4756-0.

[94] J. Gu, B. Sun, X. Du, J. Wang, Y. Zhuang, and Z. Wang, "Consortium blockchain-based malware detection in mobile devices," IEEE Access, vol. 6, pp. 12118-12128, 2018, doi: 10.1109/ACCESS.2018.2805783.

[95] S. B. Kotsiantis, "Supervised Machine Learning: A Review of Classification Techniques," Informatica, vol. 31, pp. 249-268, 2007, doi: 10.1007/s10462-007-9052-3.

[96] R. Beverly, S. Garfinkel, and G. Cardwell, "Forensic carving of network packets and associated data structures," Digit. Investig., vol. 8, pp. S78-S89, Aug. 2011, doi: 10.1016/j.diin.2011.05.010.

[97] H. S. Anderson, A. Kharkar, B. Filar, D. Evans, and P. Roth, "Learning to Evade Static PE Machine Learning Malware Models via Reinforcement Learning," 2018, [Online]. Available: http://arxiv.org/abs/1801.08917.

[98] M. R. Volodymyr Mnih, Koray Kavukcuoglu, David Silver, Alex Graves, Ioannis Antonoglou, Daan Wierstra, "Playing Atari with Deep Reinforcement Learning," pp. 1-9, 2013, [Online]. Available: http://arxiv.org/abs/1312.5602.

[99] S. L. \& D. H. Volodymyr Mnih, Koray Kavukcuoglu, David Silver, Andrei A. Rusu, Joel Veness, Marc G. Bellemare, Alex Graves, Martin Riedmiller, Andreas K. Fidjeland, Georg Ostrovski, Stig Petersen, Charles Beattie, Amir Sadik, Ioannis Antonoglou, Helen 
1130

1131

1132

1133

1134

1135

1136

1137

1138

1139

1140

1141

1142

1143

1144

1145

1146

1147

1148

1149

1150

1151

1152

1153

1154

1155

1156

1157

1158

1159

1160

1161

1162

1163

1164

1165

1166

1167

1168

1169

1170

1171

1172

1173

1174

1175

King, Dharshan Ku, "Human-level control through deep reinforcement learning," Nature, vol. 518, no. 7540, pp. 529-533, 2015, doi: 10.1038/nature14236.

[100] H. S. Anderson, B. Filar, and P. Roth, "Evading Machine Learning Malware Detection," BlackHat DC, p. 6, 2017, [Online]. Available: https://www.blackhat.com/docs/us17/thursday/us-17-Anderson-Bot-Vs-Bot-Evading-Machine-Learning-Malware-Detectionwp.pdf.

[101] X. Wan, G. Sheng, Y. Li, L. Xiao, and X. Du, "Reinforcement Learning Based Mobile Offloading for Cloud-Based Malware Detection," 2017 IEEE Glob. Commun. Conf. GLOBECOM 2017 - Proc., vol. 2018-Janua, pp. 1-6, 2018, doi: 10.1109/GLOCOM.2017.8254503.

[102] S. Mahdavifar and A. A. Ghorbani, "Application of deep learning to cybersecurity: A survey," Neurocomputing, vol. 347, pp. 149-176, 2019, doi: 10.1016/j.neucom.2019.02.056.

[103] M. Abadi, A. Agarwal, and P. Barham, "TensorFlow: Large-Scale Machine Learning on Heterogeneous Distributed Systems," 2016, [Online]. Available: http://arxiv.org/abs/1603.04467.

[104] S. K. Yangqing Jia, Evan Shelhamer, Jeff Donahue and T. D. Jonathan Long, Ross Girshick, Sergio Guadarrama, "Caffe: Convolutional architecture for fast feature embedding," MM 2014 - Proc. 2014 ACM Conf. Multimed., pp. 675-678, 2014, doi: $10.1145 / 2647868.2654889$.

[105] R. Al-Rfou, G. Alain, A. Almahairi, C. Angermueller, and D. Bahdanau, "Theano: A Python framework for fast computation of mathematical expressions," pp. 1-19, 2016, [Online]. Available: http://arxiv.org/abs/1605.02688.

[106] Y. Li, G. Wang, L. Nie, Q. Wang, and W. Tan, "Distance metric optimization driven convolutional neural network for age invariant face recognition," Pattern Recognit., vol. 75, pp. 51-62, 2018, doi: 10.1016/j.patcog.2017.10.015.

[107] R. Pascanu, J. W. Stokes, H. Sanossian, M. Marinescu, and A. Thomas, "Malware classification with recurrent networks," ICASSP, IEEE Int. Conf. Acoust. Speech Signal Process. - Proc., vol. 2015-Augus, pp. 1916-1920, 2015, doi: 10.1109/ICASSP.2015.7178304.

[108] J. Saxe and K. Berlin, "Deep neural network based malware detection using two dimensional binary program features," 2015 10th Int. Conf. Malicious Unwanted Software, MALWARE 2015, pp. 11-20, 2016, doi: 10.1109/MALWARE.2015.7413680.

[109] S. Venkatraman, M. Alazab, and R. Vinayakumar, "A hybrid deep learning image-based analysis for effective malware detection," J. Inf. Secur. Appl., vol. 47, pp. 377-389, 2019, doi: 10.1016/j.jisa.2019.06.006.

[110] P. Yang, Y. Hwa Yang, B. B. Zhou, and A. Y. Zomaya, "A Review of Ensemble Methods in Bioinformatics," Curr. Bioinform., vol. 5, no. 4, pp. 296-308, 2010, doi: 10.2174/157489310794072508.

[111] T. Hastie, R. Tibshirani, and J. Friedman, Linear Methods for Classification. 2009.

[112] Y. Amit and D. Geman, "Shape Quantization and Recognition with Randomized Trees," Neural Comput., vol. 9, no. 7, pp. 1545-1588, 1997, doi: 10.1162/neco.1997.9.7.1545.

[113] L. Lu, Z. Li, Z. Wu, W. Lee, and G. Jiang, “CHEX: Statically Vetting Android Apps for Component Hijacking Vulnerabilities," in CCS Proceedings of the ACM Conference on Computer and Communications Security, Raleigh, North Carolina, USA, 2012, pp. 229240, doi: 10.1145/2382196.2382223. 
1176

1177

1178

1179

1180

1181

1182

1183

1184

1185

1186

1187

1188

1189

1190

1191

1192

1193

1194

1195

1196

1197

1198

1199

1200

1201

1202

1203

1204

1205

1206

1207

1208

1209

1210

1211

1212

1213

1214

1215

1216

1217

1218

1219

1220

1221

[114] J. Crussell, C. Gibler, and H. Chen, "Attack of the clones: Detecting cloned applications on Android markets," Lect. Notes Comput. Sci. (including Subser. Lect. Notes Artif. Intell. Lect. Notes Bioinformatics), vol. 7459 LNCS, pp. 37-54, 2012, doi: 10.1007/978-3-64233167-1_3.

[115] A. Desnos, "Android: Static Analysis Using Similarity Distance," in 2012 45th Hawaii International Conference on System Sciences, 2012, pp. 5394-5403, doi: 10.1109/HICSS.2012.114.

[116] A. Paturi, M. Cherukuri, J. Donahue, and S. Mukkamala, "Mobile Malware Visual Analytics and Similarities of Attack Toolkits," in Collaboration Technologies and Systems (CTS), San Diego, CA, USA, 2013, pp. 149-154.

[117] M. Hammad, H. Bagheri, and S. Malek, "DELDROID: An automated approach for determination and enforcement of least-privilege architecture in android," J. Syst. Softw., vol. 149, pp. 83-100, 2019, doi: 10.1016/j.jss.2018.11.049.

[118] Samsung, "Mobile Phone," 2019. https://www.samsung.com/my/mobile/ (accessed Oct. 02, 2019).

[119] Official, "Lenovo.".

[120] Official, "LG," 2019. https://www.lg.com/my (accessed Oct. 02, 2019).

[121] Official, "Sony," 2019. https://www.sony.com.my/ (accessed Oct. 02, 2019).

[122] Official, "HTC," 2019. https://www.htc.com/my-en/ (accessed Oct. 02, 2019).

[123] Official, "Asus," 2019. https://www.asus.com/my/ (accessed Oct. 02, 2019).

[124] Official, "Motorola." https://www.motorola.com/mea/home (accessed Oct. 02, 2019).

[125] A. Moser, C. Kruegel, and E. Kirda, "Limits of static analysis for malware detection," Proc. - Annu. Comput. Secur. Appl. Conf. ACSAC, pp. 421-430, 2007, doi: 10.1109/ACSAC.2007.21.

[126] I. You and K. Yim, "Malware obfuscation techniques: A brief survey," Proc. - 2010 Int. Conf. Broadband, Wirel. Comput. Commun. Appl. BWCCA 2010, pp. 297-300, 2010, doi: 10.1109/BWCCA.2010.85.

[127] A. Firdaus, "Mobile malware anomaly-based detection systems using static analysis features," University of Malaya, 2017.

[128] Android, "ProGuard." https://developer.android.com/studio/build/shrink-code (accessed Oct. 02, 2019).

[129] S. Diego, C. Kruegel, W. Robertson, F. Valeur, and G. Vigna, "Static Disassembly of Obfuscated Binaries," 2004.

[130] N. Nissim, R. Moskovitch, L. Rokach, and Y. Elovici, "Novel active learning methods for enhanced PC malware detection in windows OS," Expert Syst. Appl., vol. 41, no. 13, pp. 5843-5857, 2014, doi: 10.1016/j.eswa.2014.02.053.

[131] W. Fan, Y. Chen, Y. Liu, and F. Wu, "DroidARA: Android Application Automatic Categorization Based on API Relationship Analysis," IEEE Access, vol. 7, no. X, pp. 157987-157996, 2019, doi: 10.1109/ACCESS.2019.2948212.

[132] J. Lee, S. Lee, and H. Lee, "Screening smartphone applications using malware family signatures," Comput. Secur., vol. 52, pp. 234-249, 2015, doi: 10.1016/j.cose.2015.02.003.

[133] L. Apvrille and A. Apvrille, "Pre-filtering Mobile Malware with Heuristic Techniques," in The 2nd International Symposium on Research in Grey-Hat Hacking (GreHack), Grenoble, France, 2013, pp. 1-16.

[134] S. Y. Yerima, S. Sezer, G. McWilliams, and I. Muttik, "A New Android Malware Detection Approach Using Bayesian Classification," in IEEE 27th International

Peer) Comput. Sci. reviewing PDF | (CS-2020:10:54261:2:0:NEW 14 Mar 2021) 
1222

1223

1224

1225

1226

1227

1228

1229

1230

1231

1232

1233

1234

1235

1236

1237

1238

1239

1240

1241

1242

1243

1244

1245

1246

1247

1248

1249

1250

1251

1252

1253

1254

1255

1256

1257

1258

1259

1260

1261

1262

1263

1264

1265

1266

1267

Conference on Advanced Information Networking and Applications (AINA), Barcelona, Spain, Mar. 2013, pp. 121-128, doi: 10.1109/AINA.2013.88.

[135] K. O. Elish, X. Shu, D. Yao, B. G. Ryder, and X. Jiang, "Profiling user-trigger dependence for Android malware detection," Comput. Secur., vol. 49, no. 540, pp. 255273, 2015, doi: 10.1016/j.cose.2014.11.001.

[136] M. Grace, Y. Zhou, Q. Zhang, S. Zou, and X. Jiang, "RiskRanker: Scalable and Accurate Zero-day Android Malware Detection," in Proceedings of the 10th International Conference on Mobile Systems, Applications, and Services, Low Wood Bay, Lake District, UK, 2011, pp. 281-293.

[137] Mathworks, "What Is Deep Learning?," 2020. .

[138] J. Qiu, J. U. N. Zhang, W. E. I. Luo, and L. E. I. Pan, “A3CM : Automatic Capability Annotation for Android Malware," IEEE Access, vol. 7, pp. 147156-147168, 2019, doi: 10.1109/ACCESS.2019.2946392.

[139] L. Zhang, V. L. L. Thing, and Y. Cheng, "A scalable and extensible framework for android malware detection and family attribution," Comput. Secur., vol. 80, pp. 120-133, 2019, doi: 10.1016/j.cose.2018.10.001.

[140] Z. Xu, K. Ren, and F. Song, "Android Malware Family Classification and Characterization Using CFG and DFG," 2019 Int. Symp. Theor. Asp. Softw. Eng., pp. 4956, 2019, doi: 10.1109/TASE.2019.00-20.

[141] and G. S. Omid Mirzaeiq, Guillermo Suarez-Tangil, Jose M. de Fuentesq, Juan Tapiadorq, "AndrEnsemble : Leveraging API Ensembles to Characterize Android Malware Families," pp. 307-314, 2019.

[142] R. V. Vega and H. Quintián, "Gaining deep knowledge of Android malware families through dimensionality," vol. 00, no. 0, doi: 10.1093/jigpal/jzy030.

[143] R. V. Vega, H. Quintián, C. Cambra, N. Basurto, Á. Herrero, and J. L. Calvo-rolle, "Delving into Android Malware Families with a Novel Neural Projection Method," vol. 2019, 2019.

[144] F. Fasano, F. Martinelli, F. Mercaldo, and A. Santone, "Cascade Learning for Mobile Malware Families Detection through Quality and Android Metrics," Proc. Int. Jt. Conf. Neural Networks, vol. 2019-July, no. July, pp. 1-10, 2019, doi: 10.1109/IJCNN.2019.8852268.

[145] W. Blanc, L. G. Hashem, K. O. Elish, and M. J. Hussain Almohri, "Identifying Android Malware Families Using Android-Oriented Metrics," Proc. - 2019 IEEE Int. Conf. Big Data, Big Data 2019, pp. 4708-4713, 2019, doi: 10.1109/BigData47090.2019.9005669.

[146] N. Xie, X. Wang, W. Wang, and J. Liu, "Fingerprinting Android malware families," Front. Comput. Sci., vol. 13, no. 3, pp. 637-646, 2019, doi: 10.1007/s11704-017-6493-y.

[147] S. Turker and A. B. Can, "AndMFC: Android malware family classification framework," 2019 IEEE 30th Int. Symp. Pers. Indoor Mob. Radio Commun. PIMRC Work. 2019, pp. 38, 2019, doi: 10.1109/PIMRCW.2019.8880840.

[148] A. Atzeni, F. Diaz, A. Marcelli, A. Sanchez, G. Squillero, and A. Tonda, "Countering android malware: A scalable semi-supervised approach for family-signature generation," IEEE Access, vol. 6, pp. 59540-59556, 2018, doi: 10.1109/ACCESS.2018.2874502.

[149] H. M. Kim, J. W. Seo, H. M. Song, and H. K. Kim, “Andro-Simnet: Android Malware Family Classification Using Social Network Analysis," arXiv, pp. 1-8, 2019.

[150] and T. L. Ming Fan, Jun Liu, Xiapu Luo, Kai Chen, Zhenzhou Tian, Qinghua Zheng, "Android malware familial classification and representative sample selection via frequent 
1268

1269

1270

1271

1272

1273

1274

1275

1276

1277

1278

1279

1280

1281

1282

1283

1284

1285

1286

1287

1288

1289

1290

1291

1292

1293

1294

1295

1296

1297

1298

1299

1300

1301

1302

1303

1304

1305

1306

1307

1308

1309

1310

1311

1312

1313

subgraph analysis," IEEE Trans. Inf. Forensics Secur., vol. 13, no. 8, pp. 1890-1905, 2018, doi: 10.1109/TIFS.2018.2806891.

[151] Y. S. Sun, C. C. Chen, S. W. Hsiao, and M. C. Chen, ANTSdroid: Automatic malware family behaviour generation and analysis for Android apps, vol. 10946 LNCS, no. August 2019. Springer International Publishing, 2018.

[152] A. Martín, V. Rodríguez-Fernández, and D. Camacho, "CANDYMAN: Classifying Android malware families by modelling dynamic traces with Markov chains," Eng. Appl. Artif. Intell., vol. 74, no. June, pp. 121-133, 2018, doi: 10.1016/j.engappai.2018.06.006.

[153] K. Aktas and S. Sen, "UpDroid: Updated Android Malware and Its Familial Classification," Lect. Notes Comput. Sci. (including Subser. Lect. Notes Artif. Intell. Lect. Notes Bioinformatics), vol. 11252 LNCS, no. November 2018, pp. 352-368, 2018, doi: 10.1007/978-3-030-03638-6 22.

[154] J. Garcia, M. Hammad, and S. Malek, "Lightweight, obfuscation-Resilient detection and family identification of android malware," ACM Trans. Softw. Eng. Methodol., vol. 26, no. 3, pp. 1-29, 2018, doi: 10.1145/3162625.

[155] A. Calleja, A. Martín, H. D. Menéndez, J. Tapiador, and D. Clark, "Picking on the family: Disrupting android malware triage by forcing misclassification," Expert Syst. Appl., vol. 95, pp. 113-126, 2018, doi: 10.1016/j.eswa.2017.11.032.

[156] F. Alswaina and K. Elleithy, "Android Malware Permission-Based Multi-Class Classification Using Extremely Randomized Trees," IEEE Access, vol. 6, pp. 7621776227, 2018, doi: 10.1109/ACCESS.2018.2883975.

[157] L. Massarelli, L. Aniello, C. Ciccotelli, L. Querzoni, D. Ucci, and R. Baldoni, "Android malware family classification based on resource consumption over time (extended version)," arXiv, no. October, 2017.

[158] H. Zhou, W. Zhang, F. Wei, and Y. Chen, "Analysis of Android Malware Family Characteristic Based on Isomorphism of Sensitive API Call Graph," Proc. - 2017 IEEE 2nd Int. Conf. Data Sci. Cyberspace, DSC 2017, no. 1, pp. 319-327, 2017, doi: 10.1109/DSC.2017.77.

[159] T. Chakraborty, F. Pierazzi, and V. S. Subrahmanian, "EC2: Ensemble Clustering and Classification for Predicting Android Malware Families," IEEE Trans. Dependable Secur. Comput., vol. 17, no. 2, pp. 262-277, 2020, doi: 10.1109/TDSC.2017.2739145.

[160] J. Sedano, S. González, C. Chira, A. L. Herrero, E. Corchado, and J. R. Villar, "Key features for the characterization of Android malware families," Log. J. IGPL, vol. 25, no. 1, pp. 54-66, 2017, doi: 10.1093/jigpal/jzw046.

[161] P. Battista, F. Mercaldo, V. Nardone, A. Santone, and C. A. Visaggio, "Identification of android malware families with model checking," ICISSP 2016 - Proc. 2nd Int. Conf. Inf. Syst. Secur. Priv., no. Icissp, pp. 542-547, 2016, doi: 10.5220/0005809205420547.

[162] S. W. Hsiao, Y. S. Sun, and M. C. Chen, "Behavior grouping of Android malware family," 2016 IEEE Int. Conf. Commun. ICC 2016, 2016, doi: 10.1109/ICC.2016.7511424.

[163] A. González, Á. Herrero, and E. Corchado, "Neural visualization of android malware families," Adv. Intell. Syst. Comput., vol. 527, pp. 576-583, 2017, doi: 10.1007/978-3319-47364-2_56.

[164] B. J. Kang, S. Y. Yerima, K. McLaughlin, and S. Sezer, "N-opcode analysis for android malware classification and categorization," 2016 Int. Conf. Cyber Secur. Prot. Digit. Serv. Cyber Secur. 2016, pp. 13-14, 2016, doi: 10.1109/CyberSecPODS.2016.7502343.

Peer] Comput. Sci. reviewing PDF | (CS-2020:10:54261:2:0:NEW 14 Mar 2021) 
1314 [165] S. Malik and K. Khatter, "System call analysis of Android Malware families," Indian J.

1315

1316

1317

1318

1319

1320

1321

1322

1323

1324

1325

1326

1327

1328

1329

1330

1331

1332

1333

1334

1335

1336

1337

1338

1339

1340

1341

1342

1343

1344

1345

1346

1347

1348

1349

1350

1351

1352

1353

1354

1355

1356

1357

1358

1359
Sci. Technol., vol. 9, no. 21, 2016, doi: 10.17485/ijst/2016/v9i21/90273.

[166] J. Sedano, C. Chira, S. González, Á. Herrero, E. Corchado, and J. R. Villar, "Characterization of android malware families by a reduced set of static features," $A d v$. Intell. Syst. Comput., vol. 527, pp. 607-617, 2017, doi: 10.1007/978-3-319-47364-2_59.

[167] Y. Feng, O. Bastani, R. Martins, I. Dillig, and S. Anand, "Automated Synthesis of Semantic Malware Signatures using Maximum Satisfiability," no. March, 2017, doi: 10.14722/ndss.2017.23379.

[168] M. Aresu, D. Ariu, M. Ahmadi, D. Maiorca, and G. Giacinto, "Clustering android malware families by http traffic," 2015 10th Int. Conf. Malicious Unwanted Software, MALWARE 2015, pp. 128-135, 2016, doi: 10.1109/MALWARE.2015.7413693.

[169] Y. Li, T. Shen, X. Sun, X. Pan, and B. Mao, "Detection, classification and characterization of android malware using API data dependency," Lect. Notes Inst. Comput. Sci. Soc. Telecommun. Eng. LNICST, vol. 164, pp. 23-40, 2015, doi: 10.1007/978-3-319-28865-9 2.

[170] L. Deshotels, V. Notani, and A. Lakhotia, "DroidLegacy," pp. 1-12, 2014, doi: $10.1145 / 2556464.2556467$.

[171] B. Kang, B. J. Kang, J. Kim, and E. G. Im, "Android malware classification method: Dalvik bytecode frequency analysis," Proc. 2013 Res. Adapt. Converg. Syst. RACS 2013, no. October, pp. 349-350, 2013, doi: 10.1145/2513228.2513295.

[172] A. Firdaus, N. B. Anuar, M. F. A. Razak, and A. K. Sangaiah, "Bio-inspired computational paradigm for feature investigation and malware detection: interactive analytics," Multimed. Tools Appl., vol. 76, no. 280, pp. 1-37, 2017, doi: 10.1007/s11042017-4586-0.

[173] A. Firdaus, N. B. Anuar, A. Karim, and M. F. A. Razak, "Discovering optimal features using static analysis and a genetic search based method for Android malware detection," Front. Inf. Technol. Electron. Eng., vol. 19, no. 6, pp. 712-736, 2018, doi: 10.1631/FITEE.1601491.

[174] S. Badhani and S. K. Muttoo, "CENDroid-A cluster-ensemble classifier for detecting malicious Android applications," Comput. Secur., vol. 85, pp. 25-40, 2019, doi: 10.1016/j.cose.2019.04.004.

[175] S. Feldman, D. Stadther, and B. Wang, "Manilyzer: Automated Android Malware Detection through Manifest Analysis," in IEEE 11th International Conference on Mobile Ad Hoc and Sensor Systems, Philadelphia, PA, USA, 2014, pp. 767-772, doi: 10.1109/MASS.2014.65.

[176] I. Islamic and T. Minna, "Android Malware Classification Using Static Code Analysis and Apriori Algorithm Improved with Particle Swarm Optimization," in World Information and Communication Technologies (WICT), Bandar Hilir, Malaysia, 2015, pp. 123-128.

[177] Université du Luxembourg, "AndroZoo," 2018. .

[178] R. Wiśniewski, "apktool," 2010. https://ibotpeaches.github.io/Apktool/.

[179] Android, "aapt," 2013. https://androidaapt.com/.

[180] A. Desnos, “Androguard," 2012. https://github.com/androguard/androguard.

[181] "Baksmali \& Smali,” 2019. https://github.com/JesusFreke/smali/wiki (accessed Oct. 02, 2019).

[182] “Dex2jar," 2019. https://sourceforge.net/p/dex2jar/wiki/UserGuide/ (accessed Oct. 02, 2019).

Peer) Comput. Sci. reviewing PDF | (CS-2020:10:54261:2:0:NEW 14 Mar 2021) 
1360 [183] Skylot, “Jadx," 2015. https://github.com/skylot/jadx (accessed Oct. 02, 2019).

1361 [184] "Dedexer." http://dedexer.sourceforge.net/ (accessed Oct. 02, 2019).

1362

1363

1364

1365

1366

[185] and G. Á. Borja Sanz, Igor Santos, Carlos Laorden, Xabier Ugarte-Pedrero, Javier Nieves, Pablo G.Bringas, "MAMA : Manifest analysis for malware detection in android," no. October, 2013, doi: 10.1080/01969722.2013.803889.

[186] B. Sarma, N. Li, C. Gates, R. Potharaju, C. Nita-rotaru, and I. Molloy, "Android Permissions: A Perspective Combining Risks and Benefits," in SACMAT'12 Proceedings

1367

1368

1369

1370

1371

1372

1373

1374

1375

1376

1377

1378

1379

1380 of the 17th ACM symposium on Access Control Models and Technologies, New Jersey, USA, 2012, pp. 13-22.

[187] O. Yildiz and I. A. Doğru, "Permission-based Android Malware Detection System Using Feature Selection with Genetic Algorithm," Int. J. Softw. Eng. Knowl. Eng., vol. 29, no. 2, pp. 245-262, 2019, doi: 10.1142/S0218194019500116.

[188] A. K. Singh, C. D. Jaidhar, and M. A. A. Kumara, "Experimental analysis of Android malware detection based on combinations of permissions and API-calls," J. Comput. Virol. Hacking Tech., vol. 15, no. 3, pp. 209-218, 2019, doi: 10.1007/s11416-019-00332$\mathrm{z}$.

[189] T. Lei, Z. Qin, Z. Wang, Q. Li, and D. Ye, "EveDroid: Event-Aware Android Malware Detection Against Model Degrading for IoT Devices," IEEE Internet Things J., vol. 6, no. 4, pp. 6668-6680, 2019, doi: 10.1109/jiot.2019.2909745.

[190] M. Junaid, D. Liu, and D. Kung, "Dexteroid: Detecting malicious behaviors in Android apps using reverse-engineered life cycle models," Comput. Secur., vol. 59, no. June, pp. 92-117, 2016, doi: 10.1016/j.cose.2016.01.008.

1381

1382

1383

1384

1385

1386

1387

1388

1389

1390

1391

1392

1393

1394

1395

1396

1397

1398

1399

1400

1401

1402

1403

1404

1405

[191] J. Huang, X. Zhang, L. Tan, P. Wang, and B. Liang, "AsDroid: Detecting stealthy behaviors in Android applications by user interface and program behavior contradiction," Proc. - Int. Conf. Softw. Eng., no. 1, pp. 1036-1046, 2014, doi: $10.1145 / 2568225.2568301$.

[192] J. Saxe, I. Labs, K. Berlin, and I. Labs, "Deep Neural Network Based Malware Detection Using Two Dimensional Binary Program Features," 2015 10th Int. Conf. Malicious Unwanted Softw., pp. 11-20, 2015, doi: 10.1109/MALWARE.2015.7413680.

[193] Z. Yang and M. Yang, "Leak miner: Detect information leakage on android with static taint analysis," in Third World Congress on Software Engineering (WCSE), Wuhan, China, 2012, pp. 101-104, doi: 10.1109/WCSE.2012.26.

[194] D. Wu, J. Liu, Y. Sui, S. Chen, and J. Xue, "Precise static happens-before analysis for detecting UAF order violations in android," IEEE 12th Int. Conf. Softw. Testing, Verif. Validation, ICST, pp. 276-287, 2019, doi: 10.1109/ICST.2019.00035.

[195] A. Liu, C. Guo, W. Wang, Y. Qiu, and J. Xu, "Static Back-Stack Transition Analysis for Android," IEEE Access, vol. 7, pp. 110781-110793, 2019, doi:

10.1109/access.2019.2934528.

[196] A. Alotaibi, "Identifying Malicious Software Using Deep Residual Long-Short Term Memory," IEEE Access, vol. 7, pp. 163128-163137, 2019, doi:

10.1109/ACCESS.2019.2951751.

[197] J. Zhang, C. Tian, and Z. Duan, "FastDroid: Efficient taint analysis for android applications," IEEE/ACM 41st Int. Conf. Softw. Eng. Companion, ICSE-Companion, pp. 236-237, 2019, doi: 10.1109/ICSE-Companion.2019.00092.

[198] S. Alsoghyer and I. Almomani, "Ransomware detection system for android applications," Electron., vol. 8, no. 8, pp. 1-36, 2019, doi: 10.3390/electronics8080868. 
1406

1407

1408

1409

1410

1411

1412

1413

1414

1415

1416

1417

1418

1419

1420

1421

1422

1423

1424

1425

1426

1427

1428

1429

1430

1431

1432

1433

1434

1435

1436

1437

1438

[199] W. Klieber, W. Snavely, L. Flynn, and M. Zheng, "Practical precise taint-flow static analysis for android app sets," ACM Int. Conf. Proceeding Ser., 2018, doi: $10.1145 / 3230833.3232825$.

[200] A. Kumar, K. S. Kuppusamy, and G. Aghila, "FAMOUS: Forensic Analysis of MObile devices Using Scoring of application permissions," Futur. Gener. Comput. Syst., vol. 83, pp. 158-172, 2018, doi: 10.1016/j.future.2018.02.001.

[201] W. Wang, Z. Gao, M. Zhao, Y. Li, J. Liu, and X. Zhang, "DroidEnsemble: Detecting Android Malicious Applications with Ensemble of String and Structural Static Features," IEEE Access, vol. 6, pp. 31798-31807, 2018, doi: 10.1109/ACCESS.2018.2835654.

[202] R. X. Ma Zhao-hui, Chen Zi-hao, Wang Xin-ming, Nie Rui-hua, Zhao Gan-sen, Wu Jiechao, "Shikra: A behavior-based Android malware detection framework," 2017, doi: 10.1109/ICGI.2017.35.

[203] S. Pooryousef and M. Amini, "Enhancing accuracy of android malware detection using intent instrumentation," ICISSP 2017 - Proc. 3rd Int. Conf. Inf. Syst. Secur. Priv., vol. 2017-Janua, no. Icissp, pp. 380-388, 2017, doi: 10.5220/0006195803800388.

[204] S. Wu, Y. Zhang, B. Jin, and W. Cao, "Practical static analysis of detecting intent-based permission leakage in Android application," Int. Conf. Commun. Technol. Proc., vol. 2017-Octob, pp. 1953-1957, 2018, doi: 10.1109/ICCT.2017.8359970.

[205] Y. C. Chang and S. De Wang, "The concept of attack scenarios and its applications in android malware detection," Proc. - 18th IEEE Int. Conf. High Perform. Comput. Commun. 14th IEEE Int. Conf. Smart City 2nd IEEE Int. Conf. Data Sci. Syst. HPCC/SmartCity/DSS 2016, pp. 1485-1492, 2017, doi: 10.1109/HPCC-SmartCityDSS.2016.0211.

[206] S. Wu, P. Wang, X. Li, and Y. Zhang, "Effective detection of android malware based on the usage of data flow APIs and machine learning," Inf. Softw. Technol., vol. 75, pp. 1725, 2016, doi: 10.1016/j.infsof.2016.03.004.

[207] N. Nissim, R. Moskovitch, O. BarAd, L. Rokach, and Y. Elovici, "ALDROID: efficient update of Android anti-virus software using designated active learning methods," Knowl. Inf. Syst., vol. 49, no. 3, pp. 795-833, 2016, doi: 10.1007/s10115-016-0918-z.

[208] M. I. Gordon, D. Kim, J. Perkins, L. Gilham, N. Nguyen, and M. Rinard, "InformationFlow Analysis of Android Applications in DroidSafe," in Network and Distributed System Security Symposium (NDSS), San Diego, CA, 2015, no. February, pp. 8-11. 
Figure 1

The flow diagram of the study.

Each step indicates the number of articles taken from Web of Science based on identification, screening, eligibility, and analysis processes.
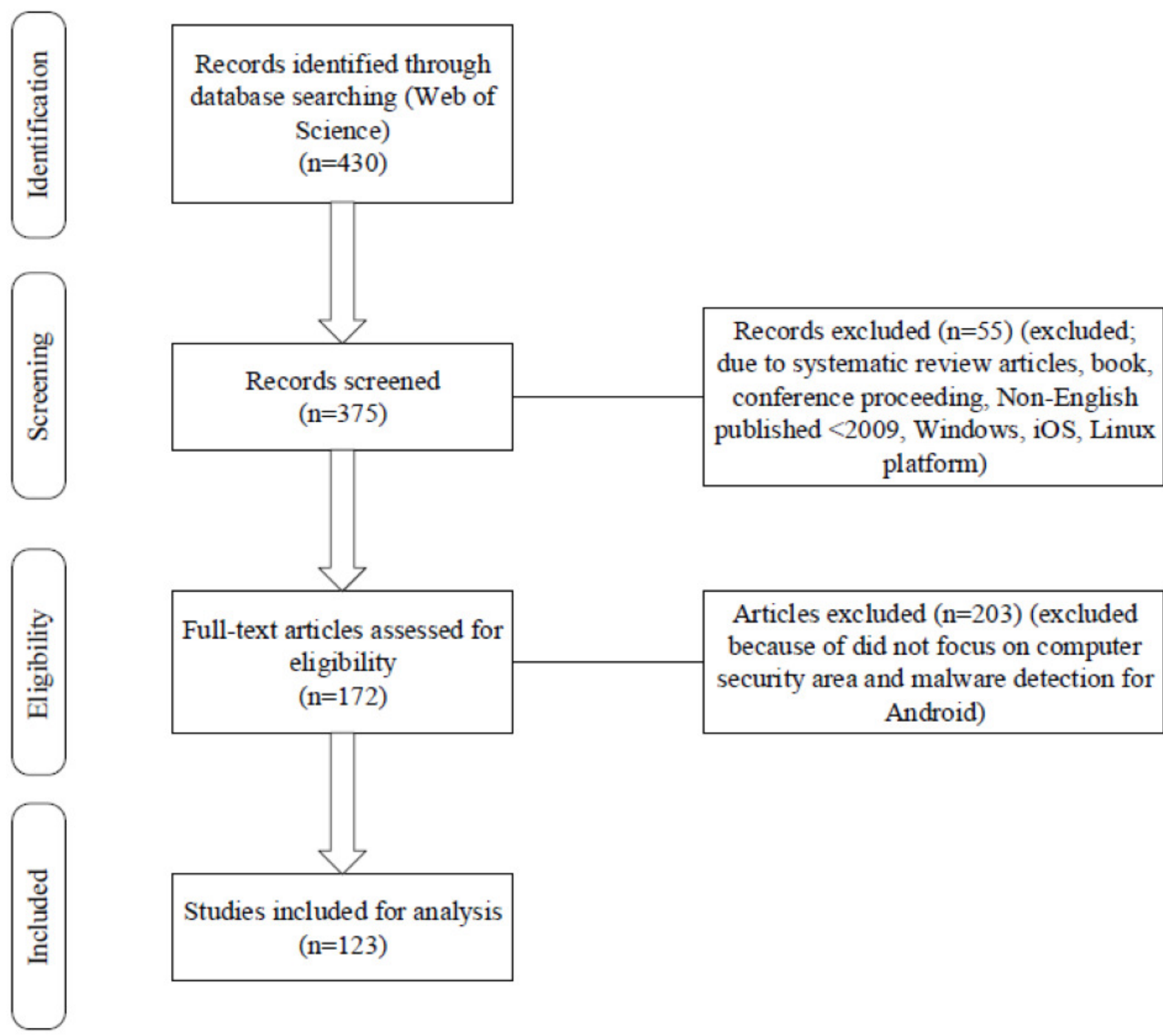

Articles excluded $(\mathrm{n}=203)$ (excluded because of did not focus on computer security area and malware detection for Android) 


\section{Figure 2}

Malware detection using static analysis.

The static analysis operation, which consisted of several steps. The steps included dataset collections, reverse engineer, features identification, and classification. 


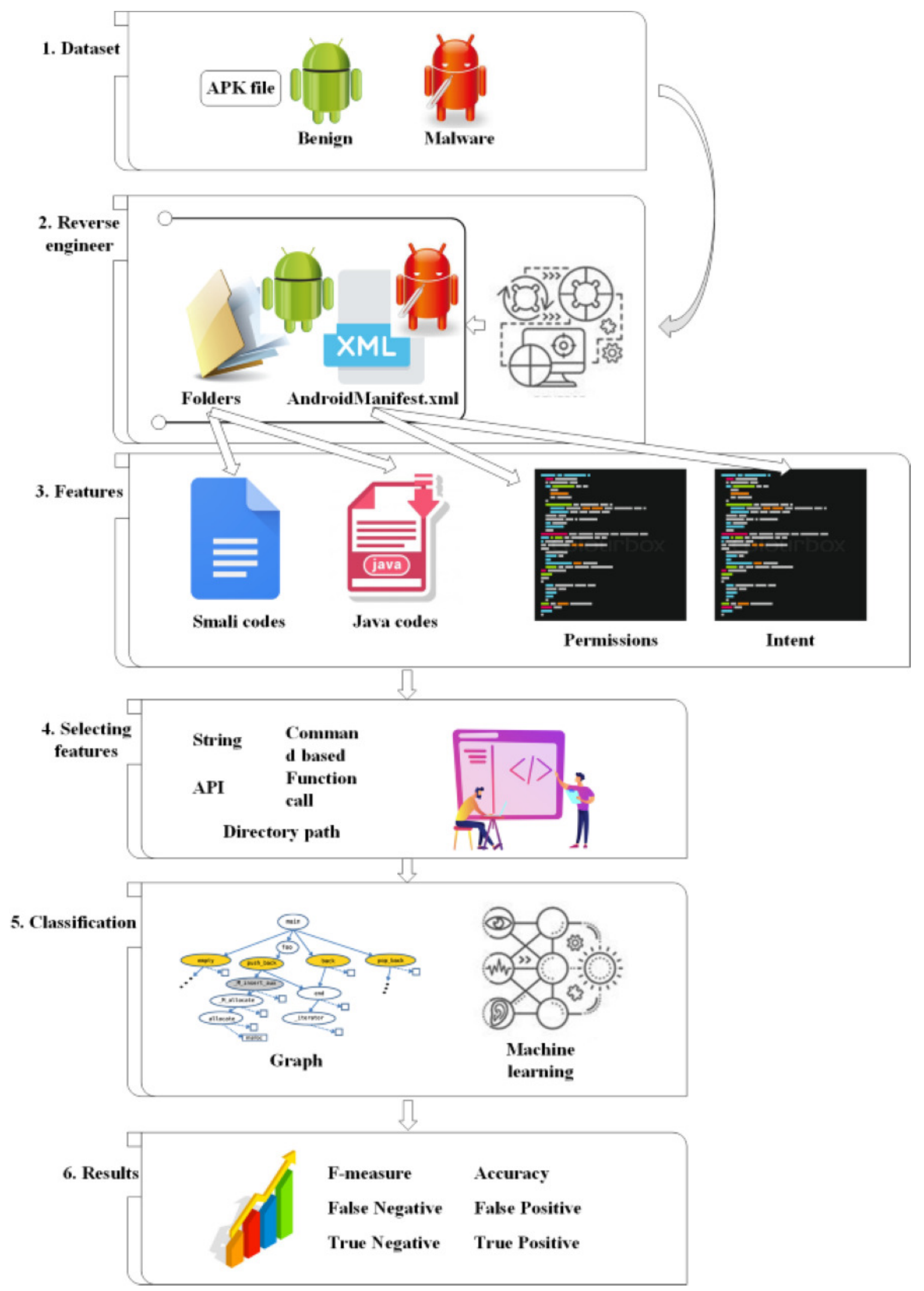


Figure 3

Dataset of Android samples.

This dataset collected from five main dataset group samples. The datasets were used by the previous researchers for their works too.

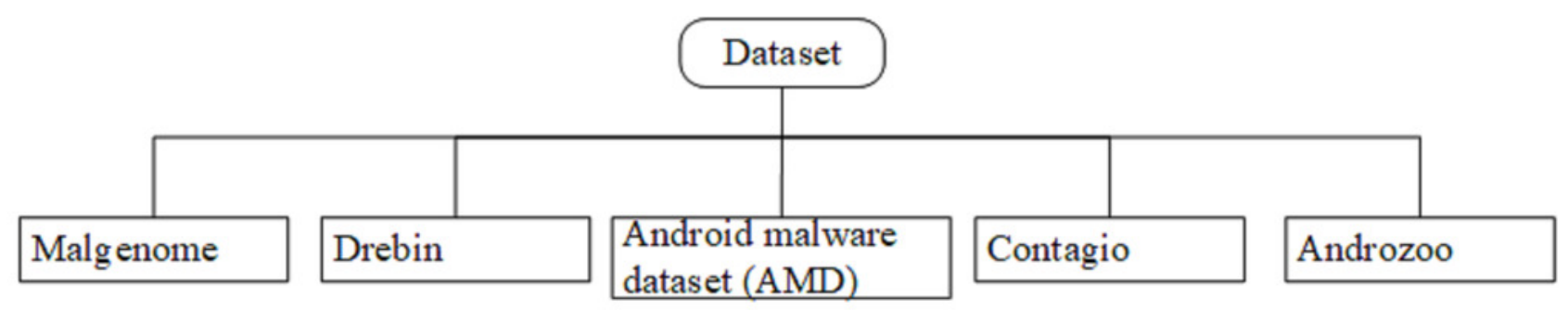


Figure 4

Reverse engineer tools for static analysis.

This is the example of reverse engineer tools that have been used by the previous researchers to extract the code for malware.

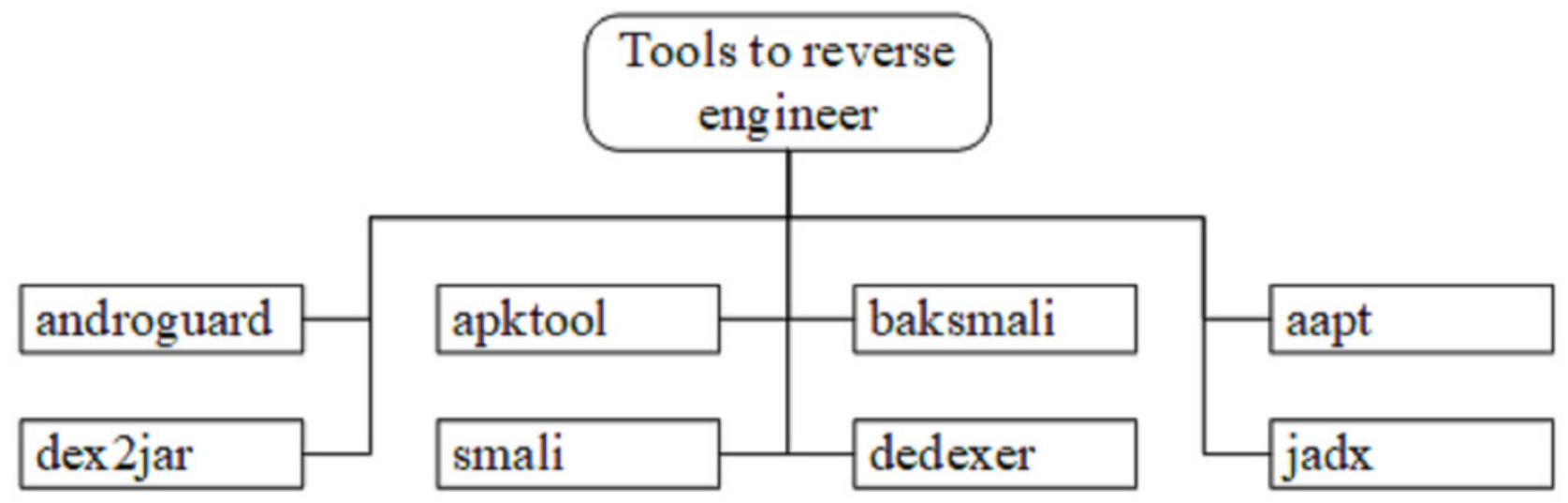


Figure 5

Taxonomy of multiple static features.

Each static feature was figure out from the various experiments done using the specific tools and methods.

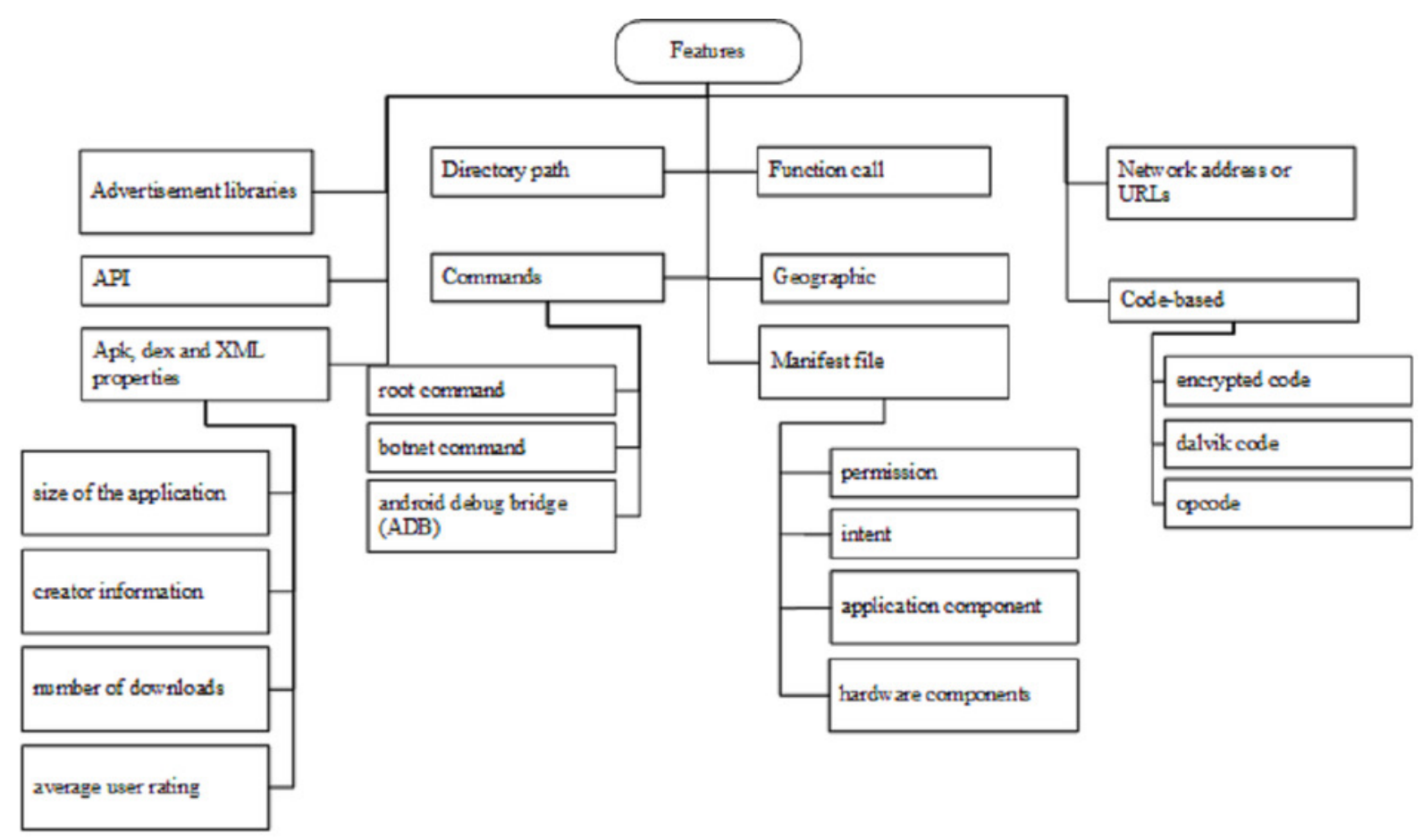


Figure 6

Categories of features in total and years.

(A) The graph shows the features identified using static analysis, depicts that researchers prefer to investigate permission and API features compare to others.(B) Total number of features based on years.

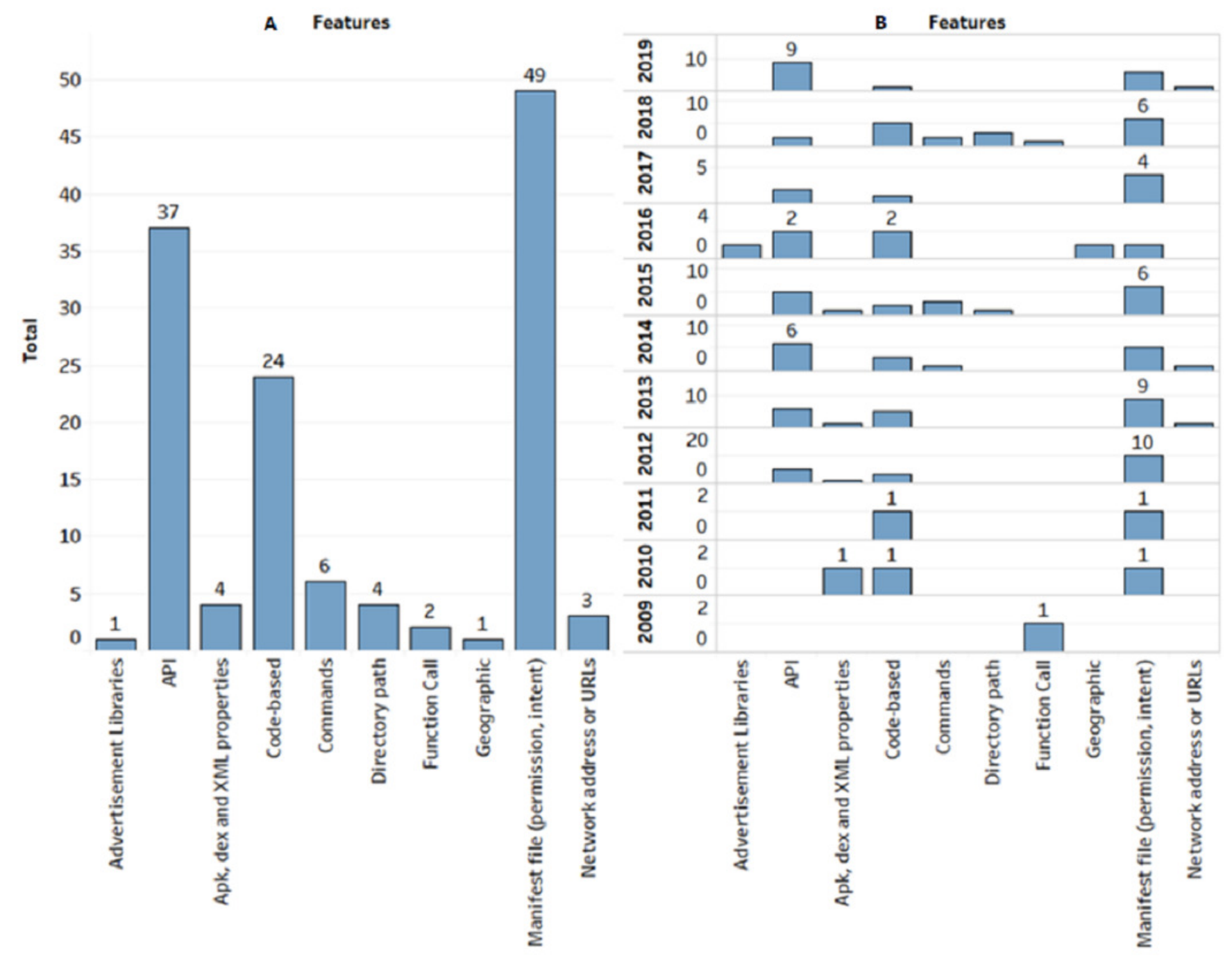


Figure 7

Mining in static analysis.

A few types of machine learning available, with each type, has its own classifier as listed in figure.

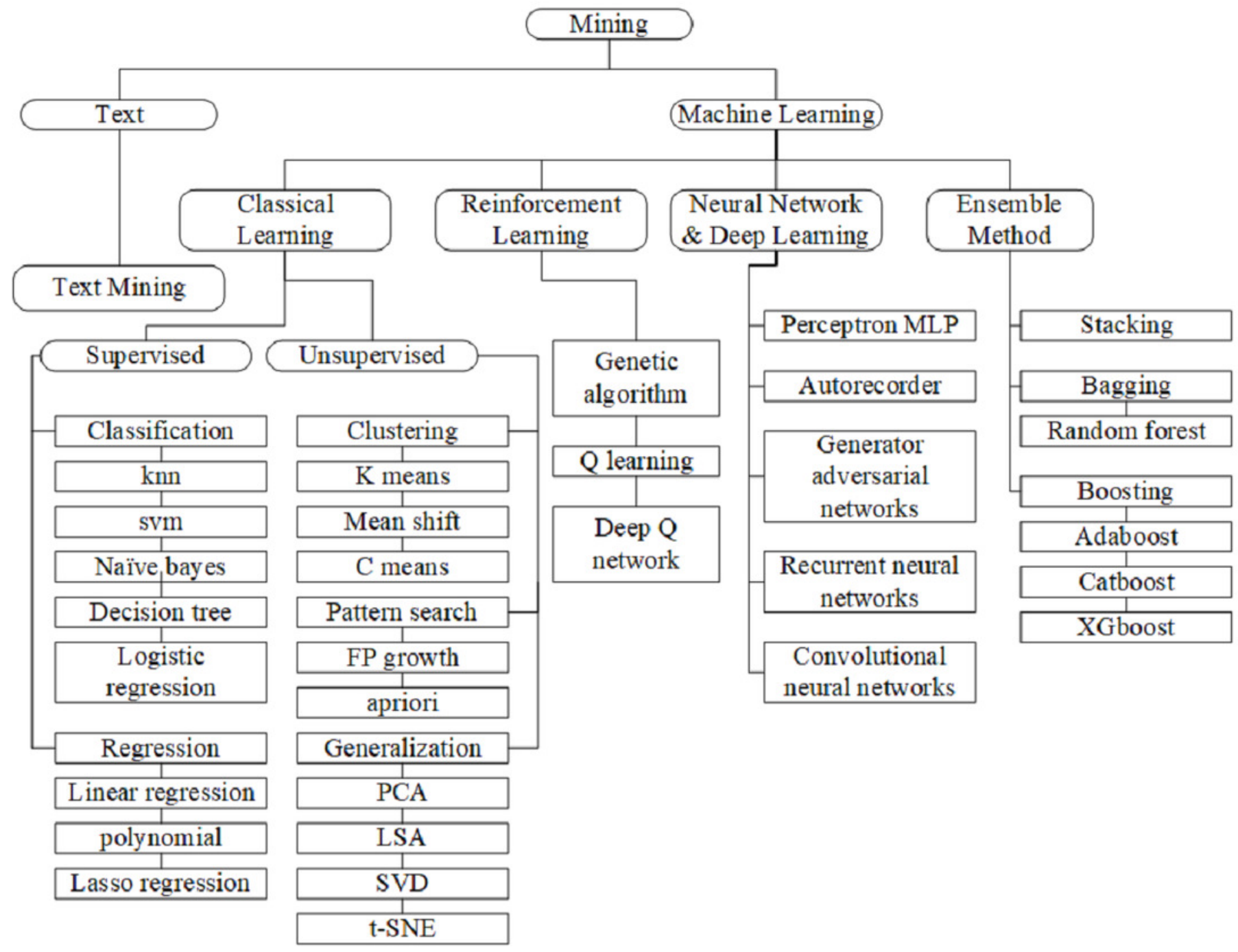


Figure 8

Differences between ML and DL.

(A) ML extract the features manually and select the ML classifier. (B) DL involves automatic feature extraction and malware classification.

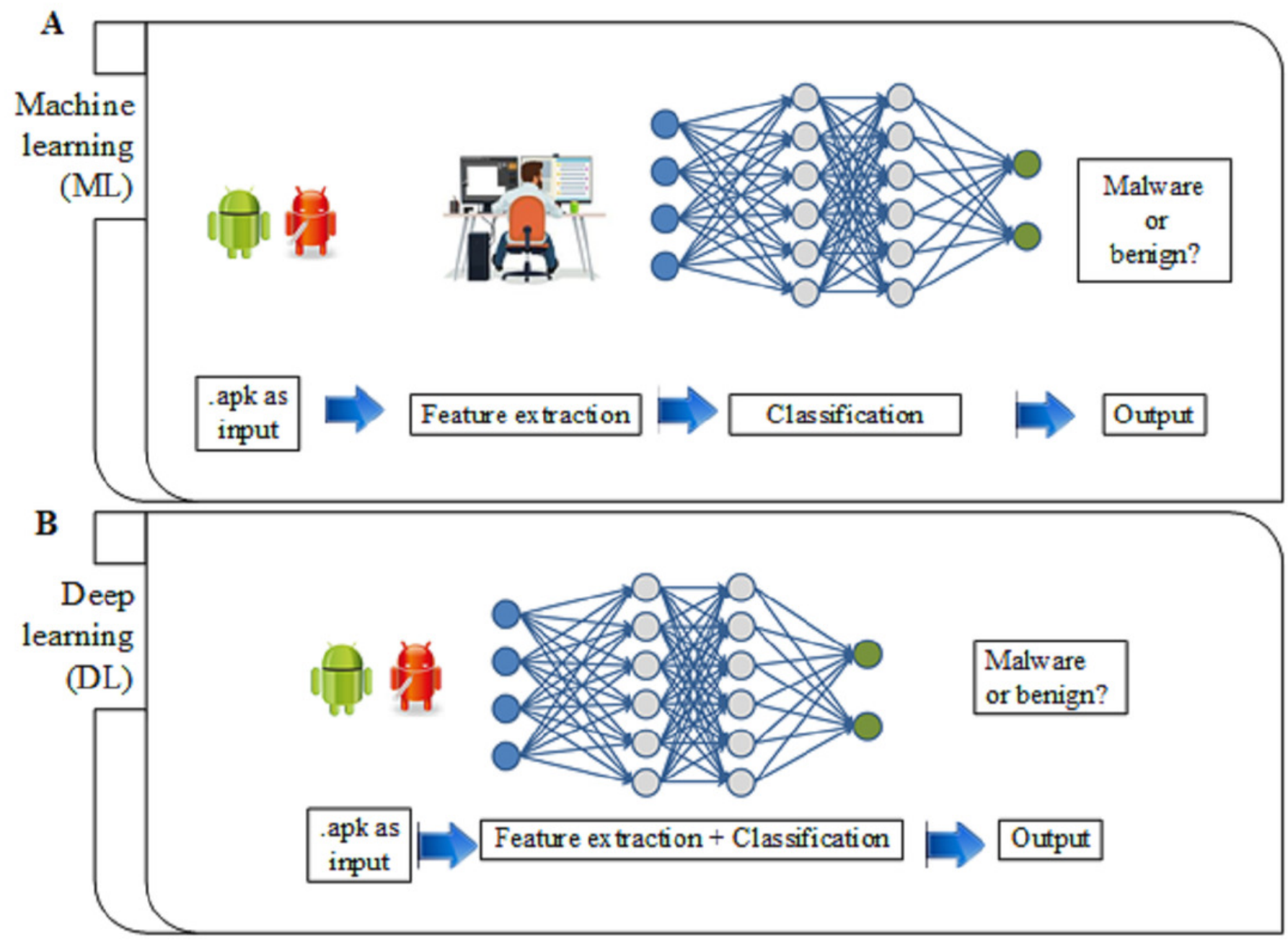


Figure 9

Popular methods among security practitioners in static analysis.

Both ML and graph were the popular methods among security practitioners in static analysis.

(A) ML was more preferred compared to graph.(B) The graph method was found to exceed the ML method in 2011, 2012, and 2014.
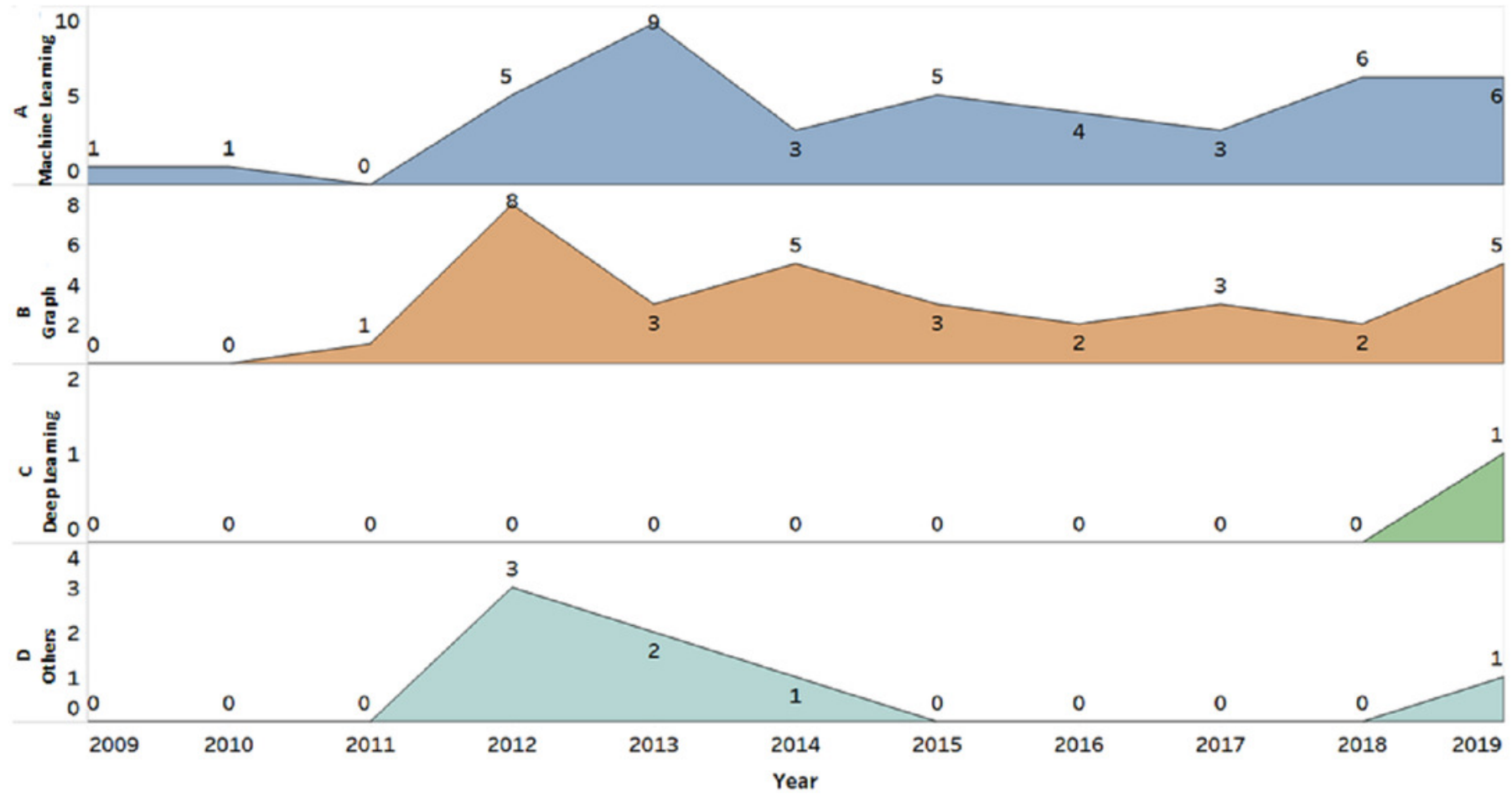


\section{Figure 10}

Types of obfuscation.

Types of obfuscation identified include encryption, oligomorphic, polymorphism, and metamorphism.

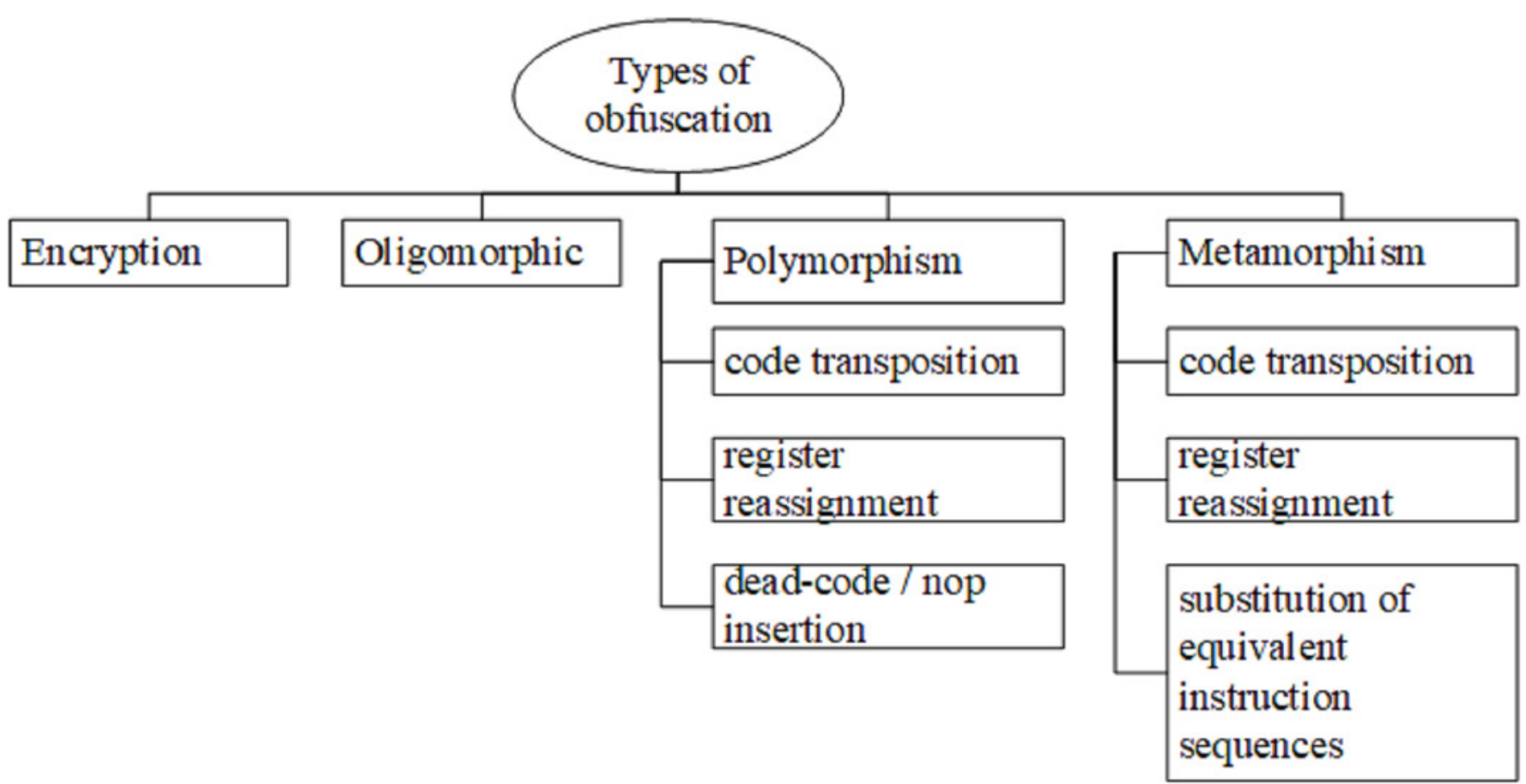




\section{Table $\mathbf{1}$ (on next page)}

Comparison malware analysis techniques.

Previous works compared using static, dynamic, and hybrid techniques. 
1 Table 1: Comparison malware analysis techniques

\begin{tabular}{|c|c|c|c|}
\hline Year & References & Analysis & Features \\
\hline 2020 & {$[16]$} & Static & Texture, color, text \\
\hline 2019 & {$[138]$} & Static & $\begin{array}{l}\text { permissions, API calls, network } \\
\text { addresses }\end{array}$ \\
\hline 2019 & {$[139]$} & Static & Assembly, Dex, Xml, Apk \\
\hline 2019 & {$[140]$} & Static & $\mathrm{CFG}, \mathrm{DFG}$ \\
\hline 2019 & {$[141]$} & Static & API calls \\
\hline 2019 & {$[142]$} & Static & Repackaging and standalone \\
\hline 2019 & [143] & Static & Root node, decision nodes, and leaf nodes \\
\hline 2019 & {$[144]$} & Static & \\
\hline 2019 & {$[145]$} & Static & Code metric \\
\hline 2019 & {$[146]$} & Static & $\begin{array}{l}\text { Platform-based permissions, hard- ware } \\
\text { components, and suspicious API calls }\end{array}$ \\
\hline 2019 & {$[147]$} & Static & Permissions and API calls \\
\hline 2018 & {$[148]$} & Hybrid & $\begin{array}{l}\text { Manifest file (i.e., number of activities, } \\
\text { permissions, receivers, filters), and the } \\
\text { source code analysis }\end{array}$ \\
\hline 2018 & {$[149]$} & Hybrid & API call \\
\hline 2018 & {$[150]$} & Static & Weighted-sensitive-API-call-based graph \\
\hline 2018 & {$[151]$} & Dynamic & $\begin{array}{l}\text { Enabling the recording of parameters and } \\
\text { return value of an API call }\end{array}$ \\
\hline 2018 & {$[152]$} & Dynamic & $\begin{array}{l}\text { transitions probabilities, states } \\
\text { frequencies, and aggregated state } \\
\text { frequencies grouped }\end{array}$ \\
\hline 2018 & {$[153]$} & Hybrid & $\begin{array}{l}\text { number of activities, services and } \\
\text { receivers given in the Manifest file and } \\
\text { the size of the APK file }\end{array}$ \\
\hline 2018 & {$[154]$} & Static & API usage, reflection-based features, and \\
\hline
\end{tabular}


features from native binaries of apps

2018

2018

2017

2017

2017

2017

2016

2016

2016

2016

2016

2016

2016

2015

2015

2015

2014

2014

2014
[155]

[156]

[157]

[158]

[159]

[160]

[161]

[162]

[163]

[150]

[164]

[165]

[166]

[167]

[132]

[168]

[169]

[170]

[171]

[86]
Static

Static

Dynamic

Static

Hybrid

Static

Static

Dynamic

Static

Static

Static

Dynamic

Static

Hybrid

Static

Dynamic

Static

Static

Static

Static
API calls, intent actions and information

flow

App's permissions

Fingerprint

API call graphs

API calls, code, Android Manifest, encryption or reflection

Minimum-Redundancy Maximum-

Relevance (MRMR)

Java Bytecode

API call

API call and the names of functions and methods

Subgraph

n-opcode feature

System call

Manifest file, apk file

Malware signatures

Signature extraction signature matching

Fine-grained HTTP structural

API data dependency

API call, apk

Bytecode frequency

Code structures

2 


\section{Table 2 (on next page)}

Comparison with previous review articles.

Summarization of previous related review articles in detecting malware. 
1 Table 1: Comparison with previous review articles

\begin{tabular}{|c|c|c|c|c|c|c|c|}
\hline References & [7] & [21] & [18] & [19] & [20] & [22] & $\begin{array}{l}\text { This } \\
\text { paper }\end{array}$ \\
\hline Title & $\begin{array}{l}\text { Investigation } \\
\text { of Static and } \\
\text { Dynamic } \\
\text { Android } \\
\text { Anti-virus } \\
\text { Strategies }\end{array}$ & $\begin{array}{l}\text { Permission- } \\
\text { based Android } \\
\text { Security: Issues } \\
\text { and } \\
\text { Countermeasures }\end{array}$ & $\begin{array}{l}\text { A } \\
\text { Review } \\
\text { on } \\
\text { Feature } \\
\text { Selection } \\
\text { in Mobile } \\
\text { Malware } \\
\text { Detection }\end{array}$ & $\begin{array}{l}\text { Securing } \\
\text { Android: A } \\
\text { Survey, } \\
\text { Taxonomy, } \\
\text { and } \\
\text { Challenges }\end{array}$ & $\begin{array}{l}\text { Android } \\
\text { Malware } \\
\text { Static } \\
\text { Analysis } \\
\text { Techniques }\end{array}$ & $\begin{array}{l}\text { A } \\
\text { Systematic } \\
\text { Literature } \\
\text { Review of } \\
\text { Android } \\
\text { Malware } \\
\text { Detection } \\
\text { Using } \\
\text { Static } \\
\text { Analysis }\end{array}$ & $\begin{array}{l}\text { Malware } \\
\text { Detection } \\
\text { using } \\
\text { Static } \\
\text { Analysis } \\
\text { for } \\
\text { Android: } \\
\text { A } \\
\text { Review } \\
\text { and Open } \\
\text { Research } \\
\text { Issue }\end{array}$ \\
\hline Year & 2013 & 2014 & 2015 & 2015 & 2015 & 2020 & $\begin{array}{l}\text { Current } \\
\text { paper }\end{array}$ \\
\hline $\begin{array}{l}\text { Citations } \\
\text { Dataset }\end{array}$ & 9 & 132 & $\begin{array}{l}172 \\
\sqrt{ }\end{array}$ & 146 & 21 & $\begin{array}{l}1 \\
\sqrt{ } \\
\sqrt{ }\end{array}$ & $\sqrt{ }$ \\
\hline $\begin{array}{l}\text { Reverse } \\
\text { engineer tools }\end{array}$ & & $\sqrt{ }$ & & & & $\sqrt{ }$ & $\sqrt{ }$ \\
\hline $\begin{array}{l}\text { All static } \\
\text { features }\end{array}$ & & & $\sqrt{ }$ & $\sqrt{ }$ & & $\sqrt{ }$ & $\sqrt{ }$ \\
\hline $\begin{array}{l}\text { All } \\
\text { classifications } \\
\text { (Machine } \\
\text { learning, deep } \\
\text { learning, } \\
\text { graph, and } \\
\text { others) }\end{array}$ & & & & & & & $\sqrt{ }$ \\
\hline $\begin{array}{l}\text { Obfuscation } \\
\text { constraints } \\
\text { and methods } \\
\text { to overcome it }\end{array}$ & & & & & & & $\sqrt{ }$ \\
\hline
\end{tabular}




\section{Table 3 (on next page)}

The main journals and conferences.

Collections of the studies that are related to Android malware detection using static analysis, where studies take up a small proportion in the primary studies. The highest number of journals and conferences are manually counted. 
1 Table 1: The main journals and conferences

\begin{tabular}{|c|c|c|}
\hline Category & Acronym & Full name \\
\hline \multirow[t]{16}{*}{ Journal } & - & IEEE Access \\
\hline & - & Computers and Security \\
\hline & PICECE & Palestinian International Conference on \\
\hline & & Electrical and Computer Engineering \\
\hline & - & Computer Virology \\
\hline & - & Expert Systems with Applications \\
\hline & ASME & Manufacturing Science and Engineering \\
\hline & KSII & Internet and Information Systems \\
\hline & - & Expert Systems with Applications \\
\hline & - & Current Bioinformatics \\
\hline & - & Neural Computation \\
\hline & - & $\begin{array}{l}\text { Frontiers of Information Technology and } \\
\text { Electronic Engineering }\end{array}$ \\
\hline & - & Neurocomputing \\
\hline & JISA & Information Security and Applications \\
\hline & - & $\begin{array}{l}\text { Advances in Intelligent Systems and } \\
\text { Computing }\end{array}$ \\
\hline & - & $\begin{array}{l}\text { IEEE Transactions on Information Forensics } \\
\text { and Security }\end{array}$ \\
\hline \multirow[t]{12}{*}{ Conference } & $\mathrm{ACM}$ & Conference on Multimedia \\
\hline & $\mathrm{DSC}$ & $\begin{array}{l}\text { IEEE International Conference on Data } \\
\text { Science in Cyberspace }\end{array}$ \\
\hline & FSE & $\begin{array}{l}\text { ACM SIGSOFT International Symposium } \\
\text { on Foundations of Software Engineering }\end{array}$ \\
\hline & TrustCom & $\begin{array}{l}\text { IEEE International Conference on Trust, } \\
\text { Security and Privacy in Computing and } \\
\text { Communications }\end{array}$ \\
\hline & GLOBECOM & IEEE Global Communications Conference \\
\hline & SIN & $\begin{array}{l}\text { International Conference on Security of } \\
\text { Information and Networks }\end{array}$ \\
\hline & MALWARE & $\begin{array}{l}\text { International Conference on Malicious and } \\
\text { Unwanted Software }\end{array}$ \\
\hline & ICC & $\begin{array}{l}\text { IEEE International Conference } \\
\text { Communications }\end{array}$ \\
\hline & ICISSP & $\begin{array}{l}\text { International Conference on Information } \\
\text { Systems Security and Privacy }\end{array}$ \\
\hline & PIMRC & $\begin{array}{l}\text { IEEE International Symposium on Personal, } \\
\text { Indoor and Mobile Radio Communications }\end{array}$ \\
\hline & IJCNN & $\begin{array}{l}\text { International Joint Conference on Neural } \\
\text { Networks }\end{array}$ \\
\hline & Big Data & IEEE International Conference on Big Data \\
\hline
\end{tabular}




\section{Table 4 (on next page)}

Advantages and disadvantages between dynamic, static and hybrid.

Dynamic, static, and hybrid analysis techniques have their own pros and cons. This table summarizes the advantages and disadvantages of these techniques. 
1 Table 1: Advantages and disadvantages between dynamic, static and hybrid

\begin{tabular}{|c|c|}
\hline Dynamic & Static \\
\hline $\begin{array}{l}\text { Able to detect unknown } \\
\text { malware }\end{array}$ & $\begin{array}{l}\text { Advantages } \\
\text { Able to detect unknown } \\
\text { malware with the aid of } \\
\text { machine learning }\end{array}$ \\
\hline \multirow{2}{*}{$\begin{array}{l}\text { Able to detect benign } \\
\text { applications, which abruptly } \\
\text { transform into malware during } \\
\text { its execution }\end{array}$} & $\begin{array}{l}\text { The application of reverse } \\
\text { engineer takes a short amount } \\
\text { of time }\end{array}$ \\
\hline & $\begin{array}{l}\text { The examination on the overall } \\
\text { code, followed by the } \\
\text { identification of a possible } \\
\text { action } \\
\text { Low resources (e.g., CPU, } \\
\text { memory, network, and storage). } \\
\text { Therefore, this analysis is } \\
\text { suitable for mobile device } \\
\text { which equipped with low } \\
\text { specifications. }\end{array}$ \\
\hline
\end{tabular}

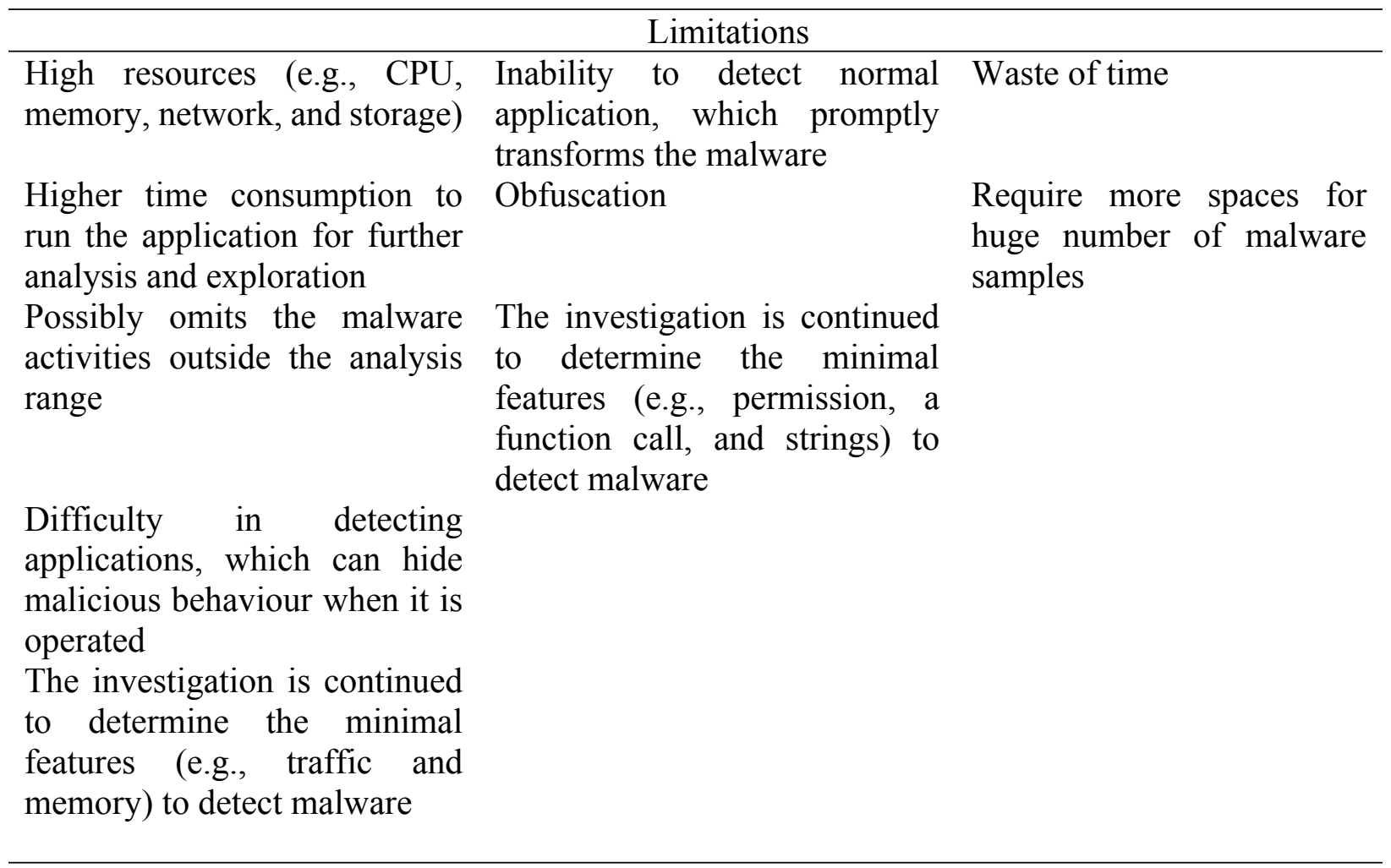




\section{Table 5 (on next page)}

Previous article with the use of different datasets.

Presents the research articles and the respective datasets, and it could be seen that the dataset providers receive significant attention from other universities and the industry. 
1 Table 1: Previous article with the use of different datasets

\begin{tabular}{ll}
\hline Dataset & $\begin{array}{l}\text { References of the articles with } \\
\text { the use of the respective } \\
\text { datasets }\end{array}$ \\
\hline Malgenome [34] & {$[13][172][65][67]$} \\
Drebin [37] & {$[172][67][173]$} \\
Android malware dataset & {$[174]$} \\
(AMD) [39] & \\
Contagio [40] & {$[175][176]$} \\
Androzoo [177] & {$[72][172][71][172]$} \\
\end{tabular}

2 


\section{Table 6(on next page)}

Previous articles and the respective reverse engineer tools.

The tools for reverse engineer adopted in the respective articles are listed in this table. 
1 Table 1: Previous articles and the respective reverse engineer tools

\begin{tabular}{ll}
\hline Tools & $\begin{array}{l}\text { References to the articles and the respective } \\
\text { tools }\end{array}$ \\
\hline apktool & {$[178][45][47][91][56]$} \\
aapt & {$[179][77]$} \\
androguard & {$[180][86][76][30][82]$} \\
baksmali & {$[181][84][75][49][51][62][44]$} \\
dex2jar & {$[182][53][59][46][56]$} \\
jadx & {$[183][65]$} \\
dedexer & {$[184]$} \\
smali & {$[171][181]$} \\
&
\end{tabular}

2 


\section{Table 7 (on next page)}

Machine learning and its classifier used in studies.

Machine learning types supervised and unsupervised with the classifier and the respective articles. To detect Android malware with static features, the supervised learning method is widely used by security practitioners. 
1 Table 1: Machine learning and its classifier used in studies

\begin{tabular}{|c|c|c|}
\hline Machine learning type & Classifier & Reference \\
\hline \multirow[t]{15}{*}{ Supervised } & K-nearest neighbor & {$[181][30]$} \\
\hline & Support & {$\left[\begin{array}{llll}{[30]} & {[76]} & {[75]} & {[55]}\end{array}\right.$} \\
\hline & machine (svm) & {$[181][182][55]$} \\
\hline & CART & [79] \\
\hline & Adaboost & {$[75][59]$} \\
\hline & Bayes & {$[13]$ [132] [73] [77] } \\
\hline & & {$[64]$} \\
\hline & Logistic Regression & {$[78]$} \\
\hline & Prism (PART) & {$[68][57][64]$} \\
\hline & $\begin{array}{l}\text { Voting feature interval } \\
\text { (vfi) }\end{array}$ & [64] \\
\hline & Random forest & {$[64][77][30][75]$} \\
\hline & $\begin{array}{l}\text { Sequential minimal } \\
\text { optimisation }(\mathrm{smo})\end{array}$ & [77] \\
\hline & $\begin{array}{l}\text { Instance-based } \\
\text { learning with } \\
\text { parameter k (ibk) }\end{array}$ & {$[77]$} \\
\hline & Simple logistic & {$[77]$} \\
\hline & Multilayer perceptron & [65] \\
\hline \multirow[t]{3}{*}{ Unsupervised } & K-means & {$[131][47][63][79]$} \\
\hline & Normalised & {$[113][114]$} \\
\hline & $\begin{array}{l}\text { Compression distance } \\
\text { (NCD) }\end{array}$ & \\
\hline
\end{tabular}




\section{Table 8(on next page)}

Static analysis works using various types of machine learning.

We can summarize classical learning is still valid to be used in experiment execution but there are a lot of works are using deep learning and graph method in the current trend. 
1 Table 1: Static analysis works using various types of machine learning

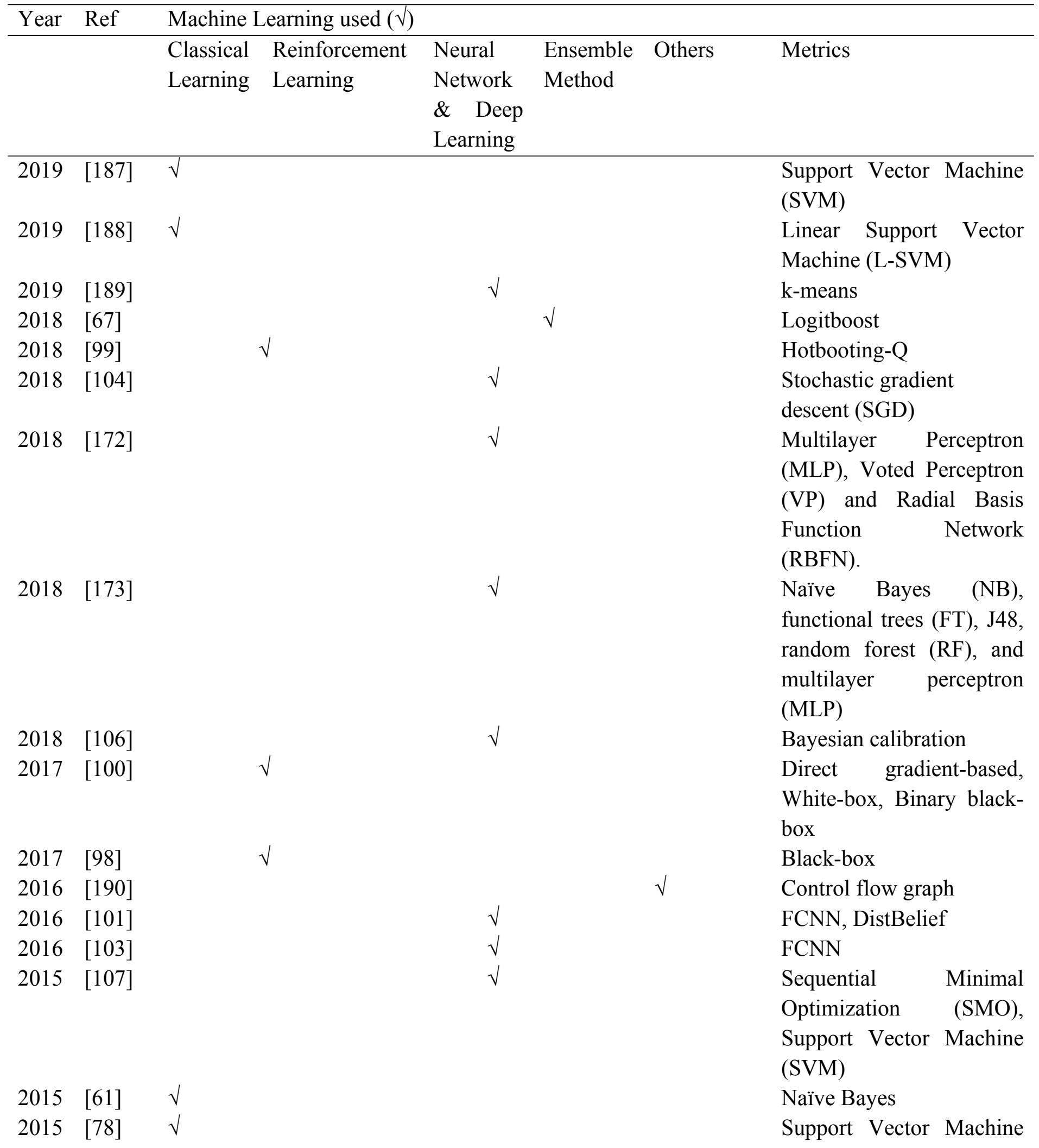




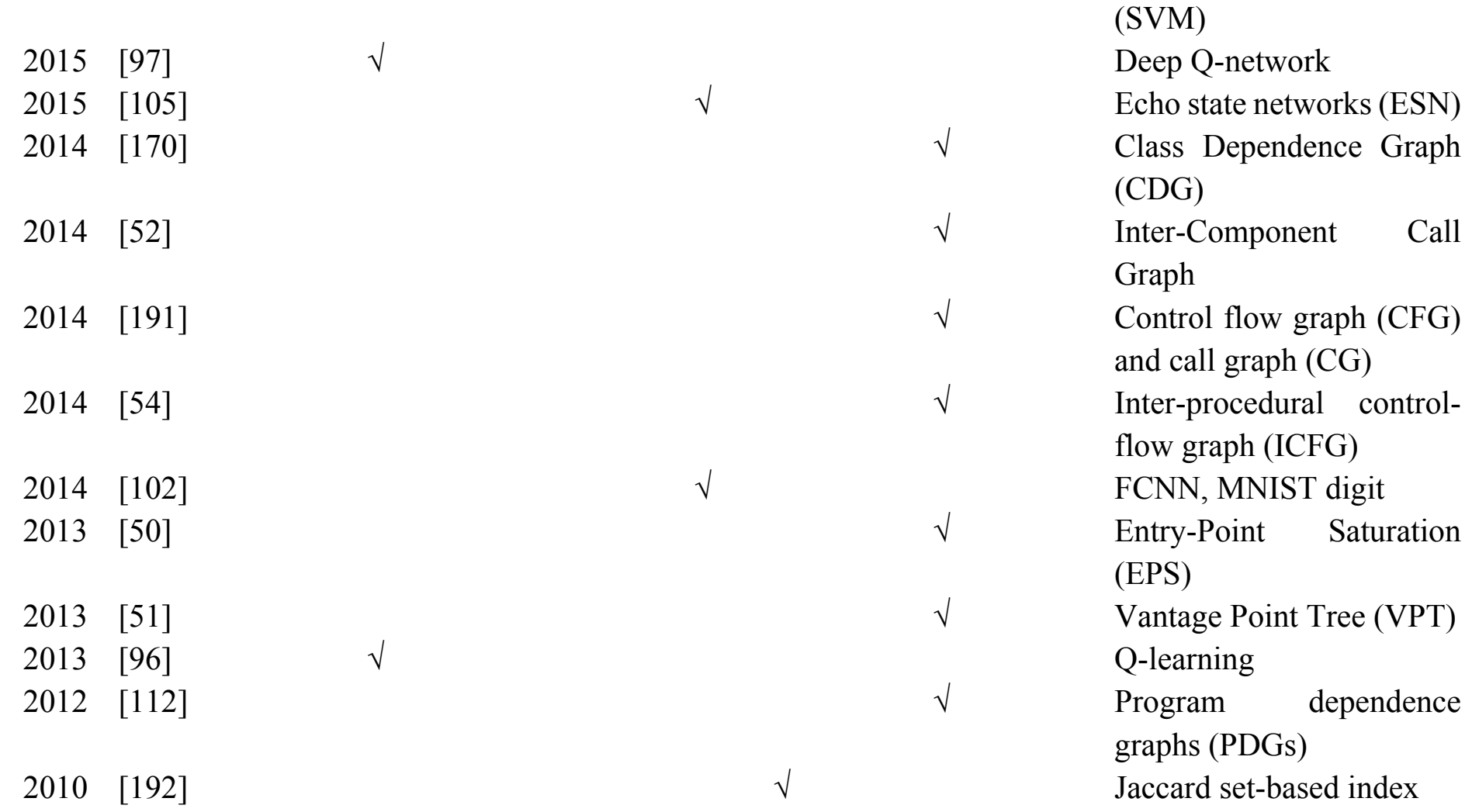




\section{Table 9 (on next page)}

Previous static analysis research, which used the graph method.

The types of analysis in graph method include call graph, inter-component call graph (ICCG), control-flow graph (CFG), and dependence graph. 
1 Table 1: Previous static analysis research, which used the graph method

\begin{tabular}{|c|c|}
\hline Type of graph & Reference \\
\hline Call graph & $\begin{array}{l}\text { Copes [48], Leakminer [193], Riskranker [136], A3 } \\
\text { [56] and [69] }\end{array}$ \\
\hline $\begin{array}{l}\text { Inter-component call } \\
\text { graph (ICCG) }\end{array}$ & [52] \\
\hline $\begin{array}{l}\text { Control flow graph } \\
\text { (CFG) }\end{array}$ & $\begin{array}{l}\text { Woodpecker [49], Flowdroid [54], Dendroid [86], } \\
\text { [76], Asdroid [53], Anadroid [50], Adrisk [44], and } \\
\text { Dexteroid [82] }\end{array}$ \\
\hline Dependency graph & $\begin{array}{l}\text { CHEX [113], Dnadroid [114], Droidlegacy [45] and } \\
\text { [51] }\end{array}$ \\
\hline
\end{tabular}

2 


\section{Table $\mathbf{1 0}$ (on next page)}

Studies conducted to overcome obfuscation.

To overcome obfuscation, many studies were conducted on di $\square$ erent approaches. 
1 Table 1: Studies conducted to overcome obfuscation

\begin{tabular}{|c|c|c|}
\hline References & Year & Solution for the obfuscation \\
\hline DNADroid [114] & 2012 & $\begin{array}{l}\text { Using programme dependence graphs } \\
\text { (PDGs), DNADroid can prevent typical } \\
\text { program transformations in obfuscation. }\end{array}$ \\
\hline [84] & 2012 & $\begin{array}{l}\text { Detects encryption attempts as one of the } \\
\text { obfuscation methods }\end{array}$ \\
\hline $\begin{array}{l}\text { DroidAPIMiner } \\
{[30]}\end{array}$ & 2013 & $\begin{array}{l}\text { Includes features, which are regularly } \\
\text { used for obfuscation, such as substring (), }\end{array}$ \\
\hline & & $\begin{array}{l}\text { indexOf(), getBytes(), valueOf(), } \\
\text { replaceAll(), Append(), getInstance(), } \\
\text { doFinal(), } \\
\text { Crypto.spec.DESKeySpec(). }\end{array}$ \\
\hline $\begin{array}{l}\text { Androsimilar } \\
\text { [91] }\end{array}$ & 2013 & $\begin{array}{l}\text { Adopts statistically strong file features } \\
\text { based on a normalised entropy }\end{array}$ \\
\hline $\begin{array}{l}\text { Droidanalytics } \\
\text { [89] }\end{array}$ & 2013 & $\begin{array}{l}\text { Only extracts the API calls in methods and } \\
\text { classes, which will be executed in the run } \\
\text { time. Additionally, the generated } \\
\text { signature, which is based on the analyst- } \\
\text { defined API, which have the ability to } \\
\text { update flexibly. }\end{array}$ \\
\hline Apposcopy [52] & 2014 & $\begin{array}{l}\text { Includes the predicate inter-component } \\
\text { communication (ICC), which allows the } \\
\text { writing of signatures, which are resilient } \\
\text { to high-level control flow obfuscation. }\end{array}$ \\
\hline Drebin [55] & 2014 & $\begin{array}{l}\text { DREBIN extracts API calls related to } \\
\text { obfuscation and loading of code, such as }\end{array}$ \\
\hline & & $\begin{array}{l}\text { DexClassLoader.loadClass }() \quad \text { and } \\
\text { Cipher.getInstance }() \text {. }\end{array}$ \\
\hline
\end{tabular}


Dendroid [86] 2014 Concentrates on the internal structure of code units (methods) to resist obfuscation.

Droidlegacy [45] 2014 Graph node represents the Java class, which detects light obfuscation.

2017 Uses Jadx (a reverse engineering tool), which provides the de-obfuscation option.

It is capable of de-obfuscating the obfuscation code in minimal error. 


\section{Table $\mathbf{1 1}$ (on next page)}

The detection of malware, which attacks Android OS, based on previous static analysis.

To identify the trends in the detection of malware through static analysis, this section presents a list of previous works of research, which cover all areas (year, features, and classi $\square$ cation). 
1 Table 1: The detection of malware, which attacks Android OS, based on previous static analysis

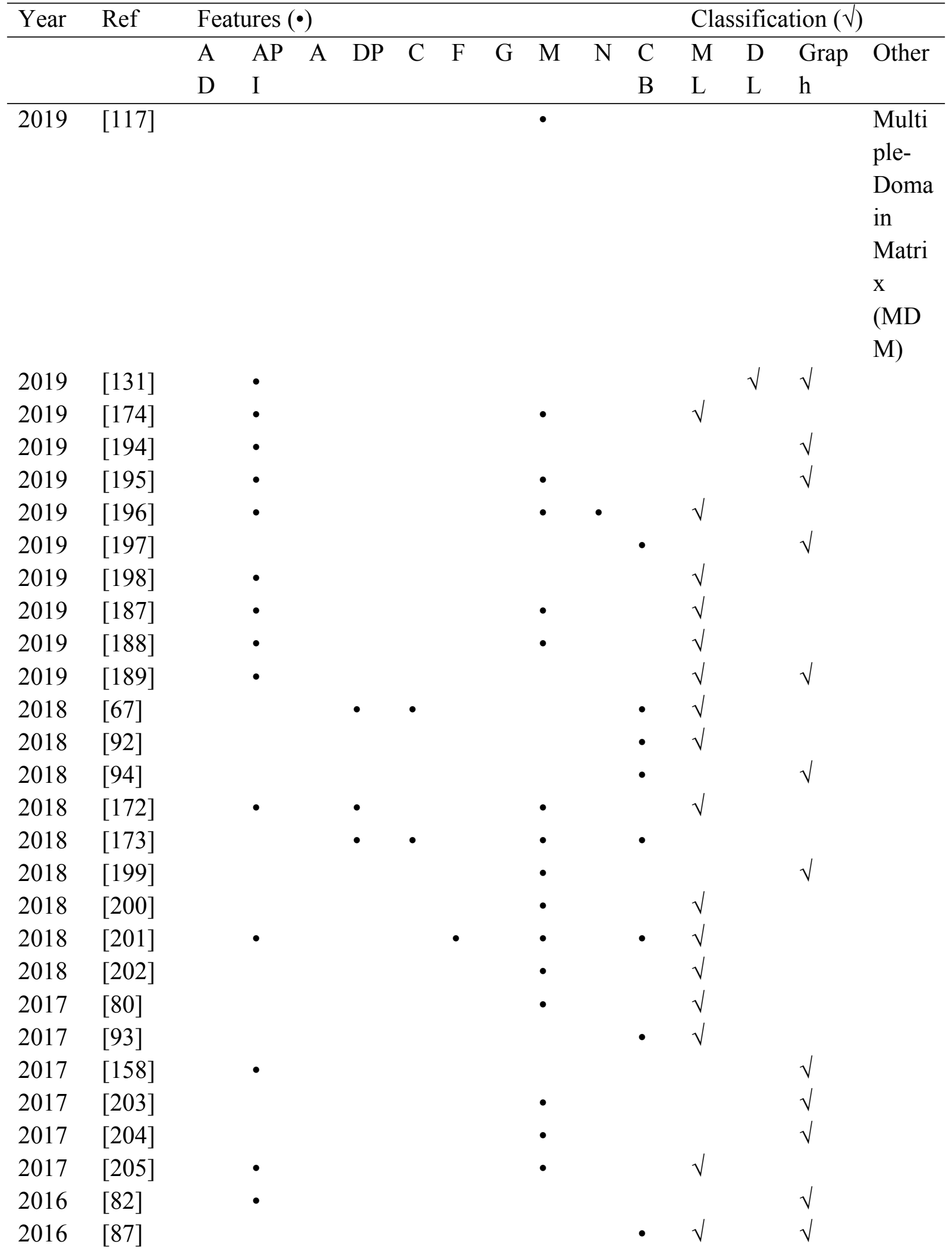




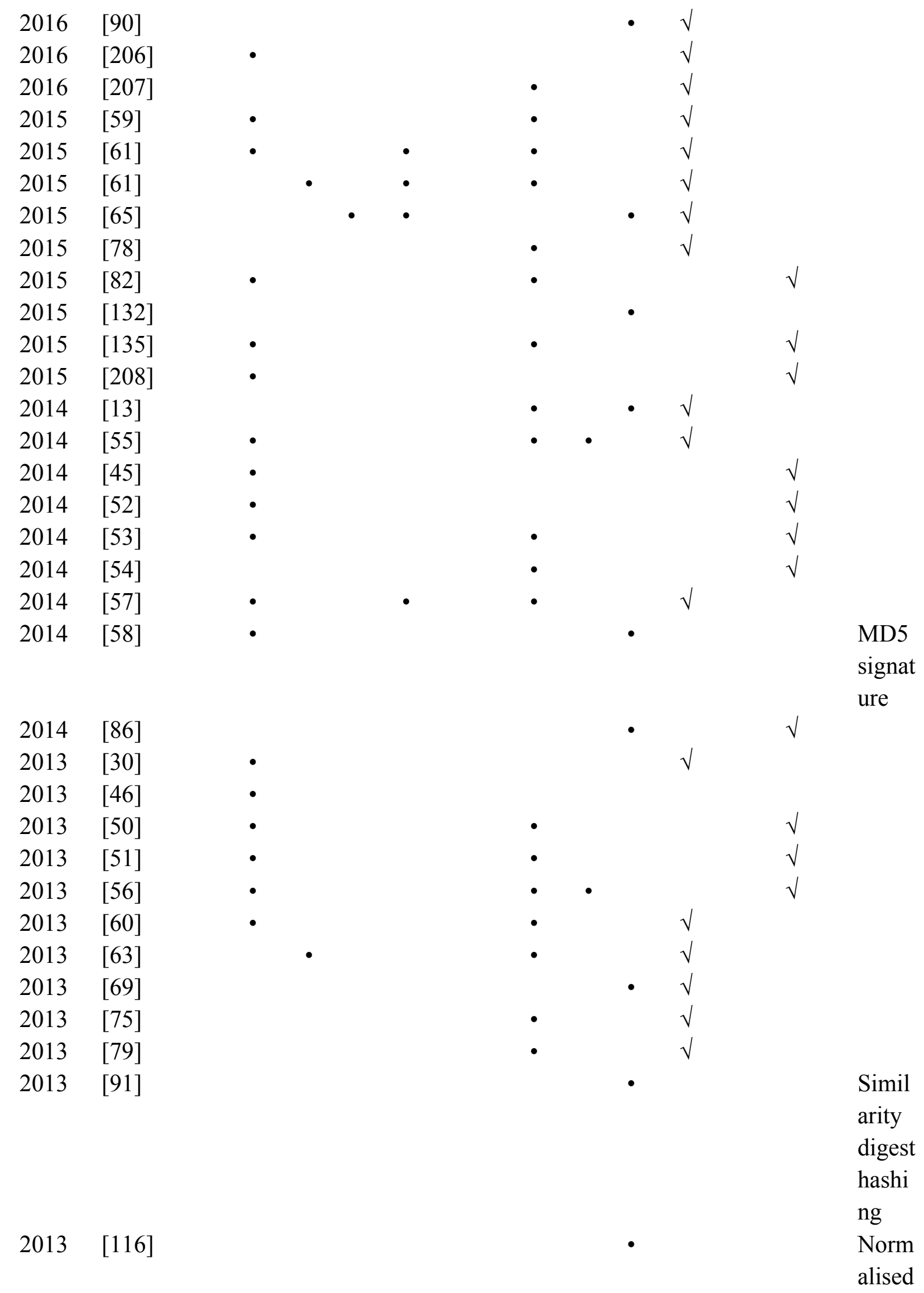




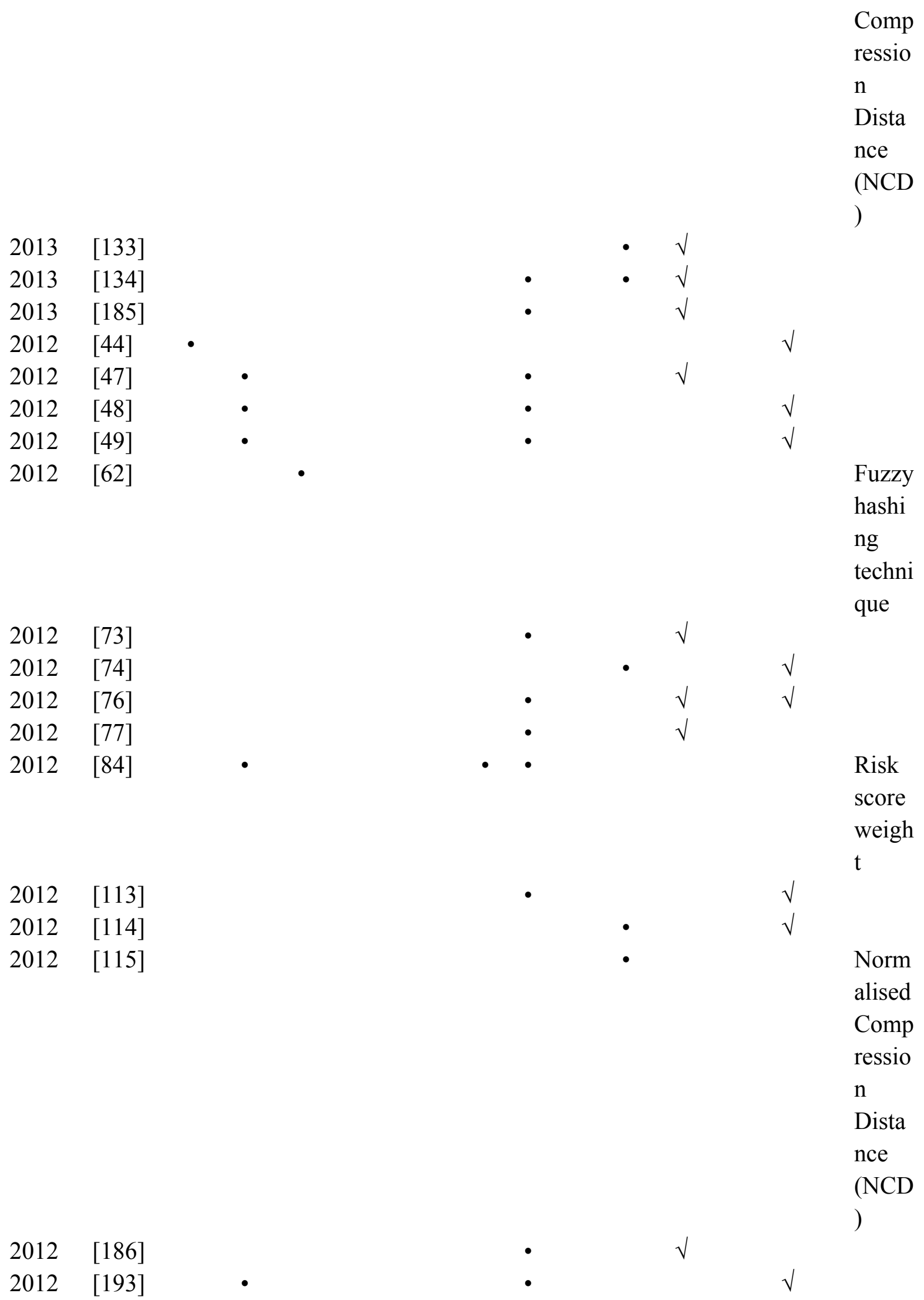




$\begin{array}{llllllll}2011 & {[136]} & & & \bullet & \bullet & & \sqrt{ } \\ 2010 & {[64]} & & & & \bullet & \bullet & \sqrt{ } \\ 2009 & {[68]} & & & & & & \sqrt{ }\end{array}$

Legends:

AD - Advertisement libraries

API - API

A - apk, dex and XML properties

DP - Directory path

C - Commands

F - Function call

G - Geographic

M - Manifest file

$\mathrm{N}$ - Network address or URLs

CB - Code-based 